\title{
Cranefly fauna (Diptera: Limoniidae, Pediciidae, Tipulidae) of the Republic of Mordovia, Russia
}

\author{
VALENTIN E. PILIPENKO ${ }^{1, \vartheta}$, ALEXANDER B. RUCHIN","v», GENNADY B. SEMISHIN ${ }^{2}$ \\ ${ }^{1}$ Lomonosov Moscow State University, GSP-1, Leninskie Gory, Moscow 119991, Russia. "email: vep@ mail.ru \\ ${ }^{2}$ Joint Directorate of the Mordovia State Nature Reserve and National Park "Smolny". Saransk, Dachnyi Lane, 4, 430011, Russia. \\ vemail: ruchin.alexander@gmail.com
}

Manuscript received: 19 November 2019. Revision accepted: 29 December 2019.

\begin{abstract}
Pilipenko VE, Ruchin AB, Semishin GB. 2020. Cranefly fauna (Diptera: Limoniidae, Pediciidae, Tipulidae) of the Republic of Mordovia, Russia. Biodiversitas 21: 355-369. The paper summarizes the Tipuloidea fauna of the Republic of Mordovia, Russia, for the first time. Among the 94 species, 29 species of Limoniidae and 14 species of Tipulidae have not been previously recorded from the region. The family Pediciidae, with five species, is also recorded for the first time. Six species are recorded from Central European Russia for the first time: Hexatoma (Hexatoma) fuscipennis (Curtis, 1836), Phylidorea (Phylidorea) bicolor (Meigen, 1804), Dicranomyia (Dicranomyia) omissinervis de Meijere, 1918, Limonia macrostigma (Schummel, 1829), Tricyphona (Tricyphona) unicolor (Schummel, 1829), and Ula (Ula) bolitophila Loew, 1869.
\end{abstract}

Keywords: Craneflies, Diptera, distribution, Limoniidae, Pediciidae, new records, Tipulidae, Mordovia, Russia

Abbreviations: Rep.: republic; MSNR: Mordovia State Nature Reserve; NPS: National Park «Smolny»; RUN: North European Russia; RUW: Northwest European Russia; RUC: Central European Russia; RUE: East European Russia; RUS: South European Russia; WS: West Siberia; ES: East Siberia; FE: Far East.

\section{INTRODUCTION}

Craneflies (Tipuloidea) comprise the families Cylindrotomidae, Limoniidae, Pediciidae and Tipulidae. The superfamily is represented by more than 16000 species in the world fauna and about 1000 in Russia (Oosterbroek 2019). Data on the cranefly fauna of European Russia are available in the works of Savchenko (Savchenko 1961, 1964, 1972, 1983, 1986, 1989). However, many common species are characterized there as "widespread in the European part," without specific regions given. Cranefly has been intensively studied recently in the European part and in other regions of Russia (Barkalov and Saaya 2014; Krivosheina 2009; Krivosheina and Krivosheina 2010, 2019; Lantsov 2009a, 2009b, 2009c, 2011a, 2011b, 2011c, 2012a, 2012b, 2012c, 2014a, 2014b, 2014c, 2015a, 2015b, 2016, 2017a, 2017b, 2017c, 2018, 2019; Lantsov and Bibin 2019; Paramonov 2006, 2011, 2012, 2014, 2016, 2017, 2018; Pilipenko 2008, 2009; Paramonov and Klepikov 2014; Paramonov and Pilipenko 2016; Polevoi and Salmela 2014; Polevoi and Pilipenko 2016; Polevoi et al. 2018; Przhiboro 2003, 2009, 2017). Craneflies of Central European Russia comprise about 200 species. The insect fauna of the Republic of Mordovia has also been intensively studied in recent years (Ruchin and Artaev 2016; Ruchin and Makarkin 2017; Ruchin and Egorov 2017, 2018a, 2018b; Ruchin 2018; Ruchin and Grishutkin 2018; Ruchin and Mikhailenko 2018; Ruchin et al. 2018; 2019a, 2019b; Tomaszewska et al. 2018; Ruchin and
Antropov 2019), including dipterans (Budaeva and Ruchin 2014; Chursina and Ruchin 2018a; 2018b; Astakhov et al. 2019). However, the fauna of Tipuloidea of Mordovia is still poorly studied. It includes only 46 species from the families Limoniidae and Tipulidae (Plavilshchikov 1964; Ruchin and Pilipenko 2015). This study significantly expands the Tipuloidea species list of Mordovia.

\section{MATERIALS AND METHODS}

\section{Study area}

The Republic of Mordovia is located in Central European Russia between $42^{\circ} 11^{\prime}$ and $46^{\circ} 45^{\prime} \mathrm{E}$ and $53^{\circ} 38^{\prime}$ and $55^{\circ} 11^{\prime} \mathrm{N}$ in the southwestern periphery of the Volga River basin between the rivers Moksha and Sura. The area includes forest and forest-steppe zones. It borders on the Chuvashia, Ryazan region, Nizhniy Novgorod region, Ulyanovsk region and Penza region (Figure 1). The eastern part of Mordovia is located in the northwest of the Volga Upland and its western part in the Oka-Don lowland. Consequently, varieties of habitats are present in the study area. In the west, north-west, and north of the country there are common boreal coniferous and mixed forests under protection of the Mordovia State Nature Reserve. Broadleaved forests cover the central and eastern parts. Forest-steppe landscapes predominate in the east and south-east (Yamashkin 1998). 


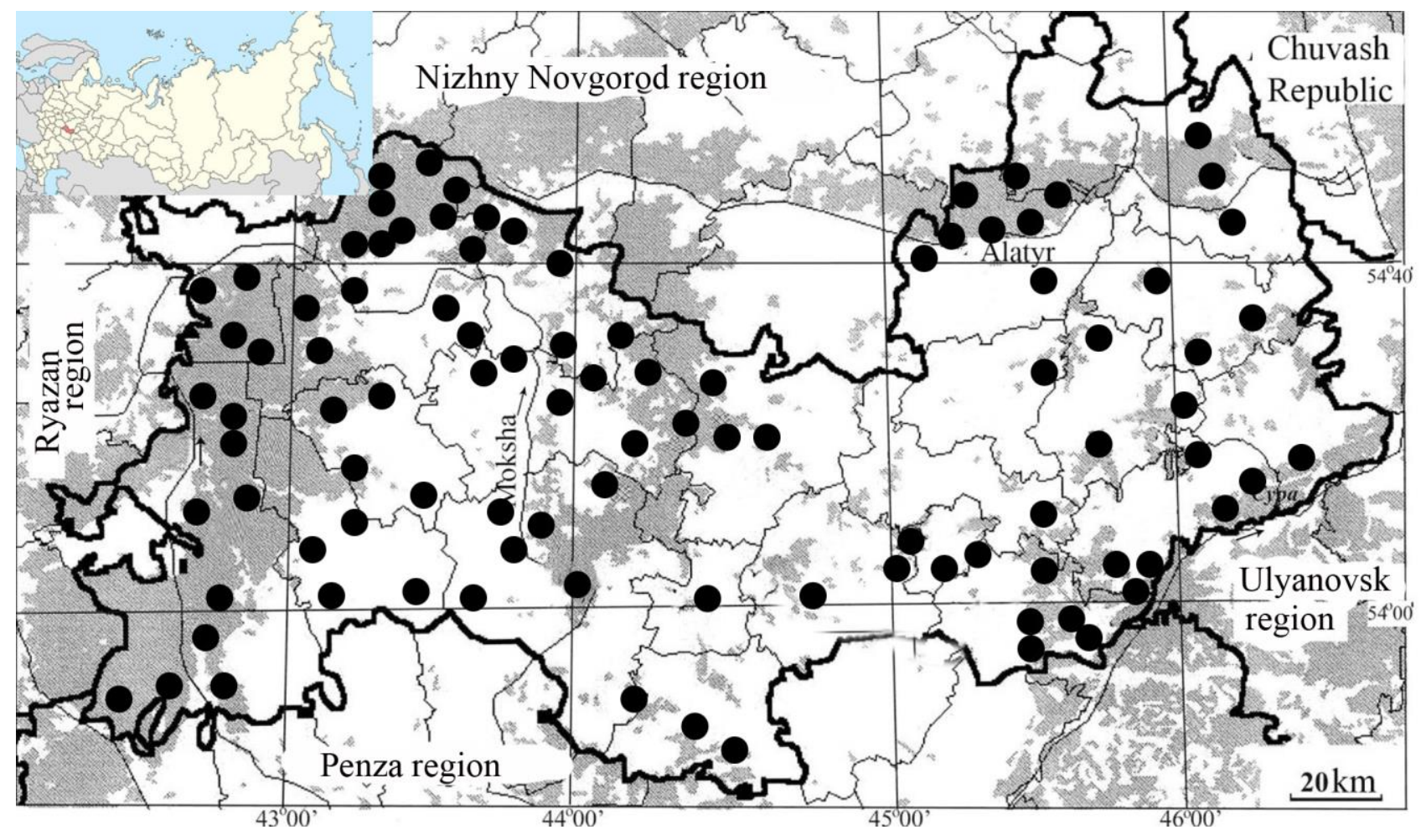

Figure 1. Study sites of cranefly in the Republic of Mordovia, Russian Federation in 2014-2018 (black dots)

The material was collected in 2014-2018 by two authors, A.B. Ruchin and G.B. Semishin (abbreviated below as RA and SG) using standard methods (Fasulati 1971) and part of the material was collected by fermenting bait traps in canopies of various, mainly deciduous trees at the height of 5-10 m (Champlain and Knull 1932; Egorov and Ivanov 2018; Makarkin and Ruchin 2018). Over 800 collected specimens were examined and identified by V.E. Pilipenko.

Specimens were studied with an Olympus SZ61 stereomicroscope. Genitalia were macerated in warm $10 \%$ $\mathrm{KOH}$ for about one hour to remove soft tissues, and then rinsed in distilled water. Cleared genitalia was preserved in microvials filled with glycerol, in the same tube as the species. A Nikon d7000 digital camera equipped with Tamron 70-300 /4-5,6 and EL-Nikkor 50/2,8 lenses and a Mitutoyo M Plan Apo 10X Microscope objective lens were used to capture stacked images, which were then combined using the Helicon Focus software (http://www.heliconsoft.com/heliconsoft-products/heliconfocus).

Morphological terminology generally follows that of McAlpine (1981). The nomenclature of taxa and distribution of species are given according to Oosterbroek (2019). The exact localities in Mordovia are given only for the newly studied material. Most of the specimens are deposited in alcohol at the Department of Entomology, Faculty of Biology of Moscow State University, Moscow.

The species newly registered in Mordovia are marked by asterisk $(*)$

\section{RESULTS AND DISCUSSION}

A total of 94 species of Tipuloidea, belonging to Limoniidae, Pediciidae and Tipulidae, were recorded in the present study. 29 species of Limoniidae and 14 species of Tipulidae are recorded in the Republic of Mordovia for the first time. The family Pediciidae, with five species, is also recorded for the first time. Six species, including Hexatoma (Hexatoma) fuscipennis (Curtis, 1836), Phylidorea (Phylidorea) bicolor (Meigen, 1804), Dicranomyia (Dicranomyia) omissinervis de Meijere, 1918, Limonia macrostigma (Schummel, 1829), Tricyphona (Tricyphona) unicolor (Schummel, 1829), Ula (Ula) bolitophila Loew, 1869 have not been previously recorded in Central European Russia. In addition to the species new for the regional fauna, findings of several rare, insufficiently known species such as Cheilotrichia neglecta (Lackschewitz), Dicranomyia schineriana (Alexander), Dicranomyia danica Kuntze, Limonia albifrons (Meigen), Ctenophora fastuosa Loew, Tipula mellea Schummel, Tipula recticornis Schummel, Tipula jutlandica Nielsen, Tipula laetibasis Alexander, Tipula winthemi Lackschewitz were highly interesting. Photographs were given for the species Dicranomyia omissinervis de Meijere, Elephantomyia krivosheinae Savchenko and Limonia nigropunctata nigropunctata (Schummel). The most abundant species in the studied material were Phylidorea ferruginea (Meigen), Dicranomyia tristis (Schummel), Metalimnobia bifasciata (Schrank), Metalimnobia quadrimaculata (Linnaeus), Tipula vernalis Meigen, 
Tipula luteipennis luteipennis Meigen, Tipula subcunctans Alexander. The fauna of the Tipuloidea of the Republic of Mordovia includes widespread Transpalaearctic (42\%), Euro-Siberian (16\%), European (20\%), Euro-West Asian $(11 \%)$ and Holarctic species $(11 \%)$.

\section{Order Diptera Linnaeus, 1758}

Family Limoniidae Speiser, 1909

Subfamily Chioneinae Rondani, 1841

*Cheilotrichia (Empeda) neglecta (Lackschewitz, 1927)

Material. Ichalki Distr., NPS, Lvovskoe forestry, post Obrezki, 18-20.IX.2017, 1ठ̄, (SG).

Distribution. Known from several European countries, including Czech Rep., Finland, Germany, Latvia, Lithuania, Norway (south), Poland, Romania, Slovakia, Switzerland. Also Kazakhstan (east); Russia: RUN (Karelia), RUC (Yaroslavl region).

\section{Erioconopa trivialis (Meigen, 1818)}

Literature. Plavilshchikov 1964; Ruchin and Pilipenko 2015.

Material. Temnikov Distr., MSNR, post Steklyannyi,

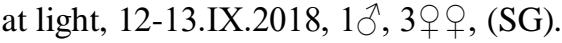

Distribution. Widely distributed in Europe, also in Georgia, Armenia, Azerbaijan, Turkey (Asiatic part), Iran; Russia: RUN, RUW, RUC (Moscow region, Rep. of Mordovia), RUE (Bashkortostan Rep.), North Caucasus.

*Erioptera (Erioptera) fuscipennis Meigen, 1818

Material. Ichalki Distr., NPS, Lvovskoe forestry, post Obrezki, 18-20.IX.2017, 1ठ̄, (SG).

Distribution. Widely distributed in Europe, also in Morocco, Algeria; Azerbaijan, Iran; Russia: RUW, RUC (Moscow region), North Caucasus (Dagestan).

\section{*Erioptera (Erioptera) lutea lutea Meigen, 1804}

Material. Ichalki Distr., NPS, Lvovskoe forestry, post Obrezki, 18-20.IX.2017, 1ð, 3우, (SG); 11-14.IX.2017, 1§, 1ㅇ, (SG); 24-30.V.2018, 3우, (SG); 16-17.VIII.2018, $3 \widehat{\jmath}, 2$ 우, (SG); 20-23.VIII.2018, 3 우, (SG); Temnikov Distr., MSNR, post Steklyannyi, at light, 17.V.2018, 1, (SG); 12-13.IX.2018, 2ðへ, (SG); Temnikov Distr., MSNR, Pushta, quarter 446, at light, 28.VIII.2018, 1ठ̄, (SG); Temnikov Distr., MSNR, Srednyaya Melnitsa, soil

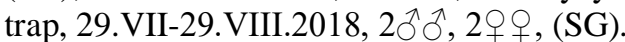

Distribution. Widely distributed throughout the Palearctic Region; Russia: RUN, RUW, RUC (Tver region, Chuvash Rep.), RUE, North Caucasus, WS (Altay, Tyva).

*Molophilus (Molophilus) ochraceus (Meigen, 1818)

Material. Temnikov Distr., MSNR, Pushta, quarter 446, at light, 28.VIII.2018, 1ठ̄, (SG).

Distribution. Widely distributed in Europe, also in Georgia, Armenia, Azerbaijan, Turkey; Russia: RUW, RUC (Tver region), RUE (Bashkortostan Rep.), North Caucasus.

*Molophilus (Molophilus) propinquus (Egger, 1863)

Material. Ichalki Distr., NPS, Lvovskoe forestry, post Obrezki, 24-30.V.2018, 1 ơ, (SG).
Distribution. Widely distributed throughout the Palearctic Region; Russia: RUN, RUW, RUE (Tatarstan Rep.), North Caucasus (Dagestan), WS (south), ES (south), FE (Primorskiy Kray, Sakhalin, Kuril Is.).

Remarks. This species is reported for the first time from Central European Russia and Mordovia. Recently, it was noted from Karelia (Humala and Polevoi 2009) and from Tatarstan Republic (Paramonov 2014).

Symplecta (Symplecta) hybrida (Meigen, 1804)

Literature. Ruchin and Pilipenko 2015.

Material. Ichalki Distr., NPS, Lvovskoe forestry, post

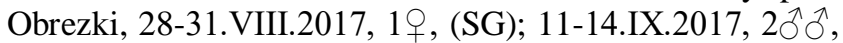
$2 \circ$ ㅇ, (SG); Ichalki Distr., NPS, sanatorium Alatyr, at light,

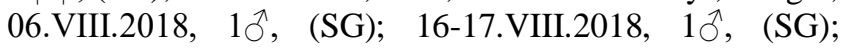
Temnikov Distr., MSNR, post Steklyannyi, at light,

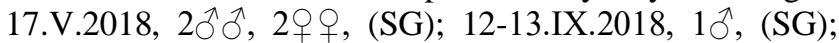
Temnikov Distr., MSNR, Srednyaya Melnitsa, soil trap, 15.V.2018, 1q, (SG).

Distribution. The species is widespread and common in the Holarctic Region; Russia: RUN, RUW, RUC (Tver and Ulyanovsk regions, Chuvash Rep., Rep. of Mordovia), RUE, North Caucasus, WS, ES (incl. Kotelnyi Is.), FE.

\section{Subfamily Limnophilinae Osten Sacken, 1869}

Dicranophragma (Brachylimnophila) nemorale (Meigen, 1818)

Literature. Ruchin and Pilipenko 2015.

Material. Temnikov Distr., MSNR, post Steklyannyi, at light, 12-13.IX.2018, 10̄, (SG).

Distribution. Widely distributed throughout the Palearctic Region; Russia: RUN, RUC (Tver region, Rep. of Mordovia), RUE, North Caucasus, WS (Altay), ES (south), FE (Primorskiy Kray, Sakhalin, Kuril Is.).

*Eloeophila maculata (Meigen, 1804)

Material. Ichalki Distr., NPS, Lvovskoe forestry, post Obrezki, 24-30.V.2018, 1 , , (SG).

Distribution. Widely distributed in Europe, also in Georgia, Armenia, Azerbaijan, Turkey (Asiatic part) and Kazakhstan (northwest); Russia: RUN (Karelia), RUW, RUC, North Caucasus.

\section{Epiphragma (Epiphragma) ocellare (Linnaeus, 1760)}

Literature. Ruchin and Pilipenko 2015.

Material. Temnikov Distr., MSNR, quarter 368, 27.V.2018, 1 , , (RA); Temnikov Distr., MSNR, quarter 403, 26.V.2016, 1, (RA); Temnikov Distr., MSNR, Pushta, at light, 26.V.2018, 1§̂, (RA).

Distribution. The species is widespread and common in the Holarctic Region; Russia: RUN, RUW, RUC (Tver, Yaroslavl and Voronezh regions, Chuvash Rep., Rep. of Mordovia), RUE, North Caucasus, WS (south), ES (south), FE (Primorskiy Kray, Kuril Is.).

*Euphylidorea (Euphylidorea) phaeostigma (Schummel, 1829)

Material. Temnikov Distr., MSNR, quarter 383, 15.V.2016, 1今, (RA). 
Distribution. Widely distributed in Europe, also in Turkey (Asiatic part); Russia: RUN, RUW, RUC (Tver and Yaroslavl regions), WS (Altay).

Eutonia barbipes (Meigen, 1804)

Literature. Ruchin and Pilipenko 2015.

Material. Temnikov Distr., MSNR, quarter 368, 27.V.2018, 3ð̊̄, (RA).

Distribution. Known from several of European countries, including Austria, Belgium, Czech Rep., Denmark, Finland (south), France, Germany, Hungary, Italy, Latvia, Lithuania, Netherlands, Poland, Slovakia, Sweden, Ukraine; Russia: RUN, RUC (Moscow, Tver, Ulyanovsk, Yaroslavl regions, Chuvash Rep., Rep. of Mordovia).

*Hexatoma (Hexatoma) fuscipennis (Curtis, 1836)

Material. Ichalki Distr., NPS, Lvovskoe forestry, post Obrezki, 24-30.V.2018, 1ठ, (SG).

Distribution. Distributed in Europe, also in Georgia, Turkey (Asiatic part) and Israel; Russia: RUN (Murmansk region), RUW, RUE.

Remarks. This species is reported for the first time from Central European Russia and Mordovia. Recently, it was noted for the east (RUE) of the European part of Russia from Maryi El and Udmurt Republic (Paramonov 2011; Przhiboro 2017). There is also an unpublished record from Murmansk region (first record for the north of Russia, Gavryushin in litt., 2011).

\section{Idioptera linnei Oosterbroek, 1992}

Literature. Ruchin and Pilipenko 2015.

Material. Ichalki Distr., NPS, sanatorium Alatyr, at light, 16-17.VIII.2018, 1§,, (SG); Temnikov Distr., MSNR, quarter 308, 10̂, (RA); MSNR, quarter 417, 03.VI.2016, $1 \hat{\partial}$, (RA); MSNR, quarter 418, $2 \hat{\jmath} \hat{\partial}$, (RA); MSNR, quarter 448, 11.V.2016, 1q, (RA); MSNR, post Steklyannyi, at light, 17.V.2018, 10, (SG); MSNR, Srednyaya Melnitsa, soil trap, 15.V.2018, 1, (SG).

Distribution. Widely distributed throughout the Palearctic Region; Russia: RUN, RUW, RUC (Tver region, Rep. of Mordovia), WS, ES, FE (Amur region).

\section{Idioptera pulchella (Meigen, 1830)}

Literature. Ruchin and Pilipenko 2015.

Distribution. Widely distributed throughout the Palearctic Region; Russia: RUN, RUW, RUC (Tver region, Rep. of Mordovia), RUE (Tatarstan Rep.), RUS (Volgograd region), North Caucasus (Kabardino-Balkar Rep.), WS (south), ES (south), FE (Magadan region, Amur region).

\section{Limnophila (Limnophila) pictipennis (Meigen, 1818)}

Literature. Ruchin and Pilipenko 2015.

Distribution. Widely distributed throughout the Palearctic Region; Russia: RUW, RUC (Tver region, Rep. of Mordovia), RUE (Orenburg region), North Caucasus, WS (south), ES (south), FE (Khabarovskiy Kray, Primorskiy Kray).
Limnophila (Limnophila) schranki Oosterbroek, 1992

Literature. Ruchin and Pilipenko 2015.

Material. Temnikov Distr., MSNR, quarter 408,

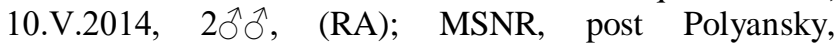
18.V.2014, 1§̂, (RA).

Distribution. Distributed in Europe, also in Georgia, Turkey (Asiatic part) and Kazakhstan (northwest); Russia: RUN, RUW, RUC (Tver, Ulyanovsk regions, Rep. of Mordovia) and North Caucasus.

*Phylidorea (Paraphylidorea) fulvonervosa (Schummel, 1829)

Material. Ichalki Distr., NPS, Lvovskoe forestry, post Obrezki, 20-23.VIII.2018, 1§̂, (SG); Temnikov Distr., MSNR, post Steklyannyi, at light, 12-13.IX.2018, 1§̄, (SG).

Distribution. Widely distributed throughout the Palearctic Region; Russia: RUN, RUW, RUC (Tver region), RUE (Bashkortostan Rep.), FE (Primorskiy Kray, Sakhalin, Kuril Is.).

*Phylidorea (Phylidorea) bicolor (Meigen, 1804)

Material. Temnikov Distr., MSNR, Pushta, at light, 13.V.2014, 1ㅇ, (RA); Temnikov Distr., MSNR, Srednyaya Melnitsa, soil trap, 15.V.2018, 1§̂, 1, (SG).

Distribution. Known from European countries, including Austria, Belgium, Czech Rep., Denmark, Estonia, Finland, France, Germany, Great Britain, Latvia, Lithuania, Norway (south), Poland, Romania, Serbia, Slovakia, Slovenia, Sweden, Ukraine; Russia: RUN, RUW, WS (Altay).

Remarks. This species is reported for the first time from Central European Russia and Mordovia.

\section{*Phylidorea (Phylidorea) ferruginea (Meigen, 1818)}

Material. Ichalki Distr., NPS, Lvovskoe forestry, post Obrezki, 11-14.IX.2017, 1ð, 4우, (SG); 18-20.IX.2017,

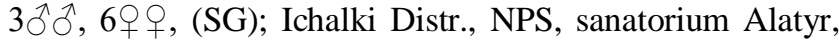
at light, 06-08.VIII.2018, 1ठ, (SG); Temnikov Distr., MSNR, quarter 368, 27.V.2018, 1क, (RA); Temnikov Distr., MSNR, post Steklyannyi, at light, 12-13.IX.2018, $10 \hat{\jmath}, 5+$ 우, (SG); MSNR, Pushta, quarter 446, at light,

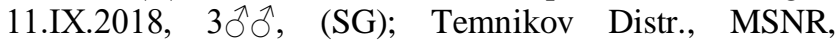
Srednyaya Melnitsa, soil trap, 29.VII-29.VIII.2018, 10, (SG).

Distribution. Widely distributed in Europe, also in Armenia, Azerbaijan, Turkey (Asiatic part), Israel and in East Palearctic: Kazakhstan, Turkmenistan, Uzbekistan, Tajikistan, Kyrgyzstan, Mongolia; Russia: RUN, RUW, RUC (Yaroslavl and Tver regions), RUE (Bashkortostan Rep.), RUS, North Caucasus, WS (Tyva).

*Phylidorea (Phylidorea) squalens (Zetterstedt, 1838)

Material. Temnikov Distr., MSNR, quarter 373, 20.VI.2015, 1ठ̀, (RA).

Distribution. Distributed in Europe, and in Mongolia; Russia: RUN, RUW, RUC (Tver region), ES (south side of Lake Baikal). 
*Pilaria discicollis (Meigen, 1818)

Material. Ichalki Distr., NPS, Lvovskoe forestry, post Obrezki, 11-14.IX.2017, 2ㅇ, (SG).

Distribution. Russia: Distributed in Europe, also in Turkey (Asiatic part), Iran; RUN (Arkhangelsk region, Karelia), RUW, RUC (Tver region), RUE.

*Pilaria fuscipennis (Meigen, 1818)

Material. Ichalki Distr., NPS, Lvovskoe forestry, post Obrezki, 20-23.VIII.2018, 1 $\odot$, (SG).

Distribution. Widely distributed throughout the Palearctic Region; Russia: RUW, RUC, North Caucasus, WS (Altay), ?FE.

\section{Subfamily Limoniinae Kuntze, 1920}

*Achyrolimonia neonebulosa (Alexander, 1924)

Material. Ichalki Distr., NPS, sanatorium Alatyr, at light, 16-17.VIII.2018, 1今, 2 우, (SG).

Distribution. The species is widespread in the Holarctic Region; Russia: RUC (Tver region), North Caucasus, WS (Altay), FE (Primorskiy Kray, Sakhalin).

*Dicranomyia (Dicranomyia) autumnalis (Staeger, 1840)

Material. Ichalki Distr., NPS, Lvovskoe forestry, post Obrezki, 11-14.IX.2017, 19, (SG); 18-20.IX.2017, 1ठ, (SG); Temnikov Distr., MSNR, post Steklyannyi, at light, 12-13.IX.2018, 2ðึ, 1q, (SG).

Distribution. Widely distributed in Europe, also in Kazakhstan (Pavlodar region) and India (Kashmir); Russia: RUN, RUW, RUC (Yaroslavl region).

*Dicranomyia (Dicranomyia) consimilis (Zetterstedt, 1838)

Material. Temnikov Distr., MSNR, post Steklyannyi, at light, 12-13.IX.2018, 1今, (SG).

Distribution. Widely distributed throughout the Palearctic Region; Russia: RUN, RUC (Tver region), RUE (Bashkortostan Rep.), ES (south), FE (Magadan region, Primorskiy Kray, Sakhalin, Kuril Is.).

\section{Dicranomyia (Dicranomyia) frontalis (Staeger, 1840)}

Literature. Ruchin and Pilipenko 2015.

Material. Ichalki Distr., NPS, sanatorium Alatyr, at light, 06-08.VIII.2018, 1ڤ̂, 1ㅇ, (SG); 16-17.VIII.2018, 1ठ, 6 오, (SG).

Distribution. Widely distributed in Holarctic and Oriental Region; Russia: RUN, RUW, RUC (Yaroslavl, Tver regions, Rep. of Mordovia), RUE (Bashkortostan Rep.), WS (south), ES (south), FE (Magadan region, Khabarovskiy Kray, Primorskiy Kray, Sakhalin, Kuril Is.).

*Dicranomyia (Dicranomyia) modesta (Meigen, 1818)

Material. Ichalki Distr., NPS, Lvovskoe forestry, post Obrezki, 28-31.VIII.2017, 1ð̄, 4우, (SG); 18-20.IX.2017,

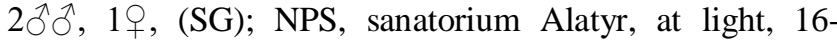
17.VIII.2018, 1ð, (SG); Temnikov Distr., MSNR, Srednyaya Melnitsa, soil trap, 29.VII-29.VIII.2018, $2 \hat{\jmath}$, (SG).

Distribution. The species is widespread in the Holarctic Region; Russia: RUN, RUW, RUC (Chuvash
Rep., Tver and Yaroslavl regions), RUE, North Caucasus, WS, ES, FE.

*Dicranomyia (Dicranomyia) omissinervis de Meijere, 1918 (Figure 2)

Material. Ichalki Distr., NPS, sanatorium Alatyr, at light, 16-17.VIII.2018, 1§̂, (SG).

Distribution. Widely distributed throughout the Palearctic Region; Russia: RUN (Arkhangelsk region), RUW, RUE (Bashkortostan Rep.), WS (Tyva), ES (Irkutsk region), FE (Magadan region, Kamchatka, Khabarovskiy Kray, Primorskiy Kray, Sakhalin, Kuril Is.).

Remarks. This species is reported for the first time from Central European Russia and Mordovia. The specimen from Mordovia shows an unusual discal cell (Figure $2 \mathrm{~A}$ ), which usually is open.

*Dicranomyia (Dicranomyia) ventralis (Schummel, 1829)

Material. Ichalki Distr., NPS, Lvovskoe forestry, post Obrezki, 18-20.IX.2017, 1ठ, (SG); NPS, sanatorium Alatyr, at light, 06-08.VIII.2018, 19, (SG); Temnikov Distr., MSNR, Pushta, quarter 446, at light, 11.IX.2018, 10, (SG); MSNR, post Steklyannyi, at light, 1213.IX.2018, $2 \hat{\jmath} \hat{\jmath},(\mathrm{SG})$

Distribution. Widely distributed throughout the Palearctic Region and India; Russia: RUN, RUW, RUC (Tver and Yaroslavl regions), RUE (Bashkortostan Rep.), WS, ES (Siberia eastward to Yakutia).
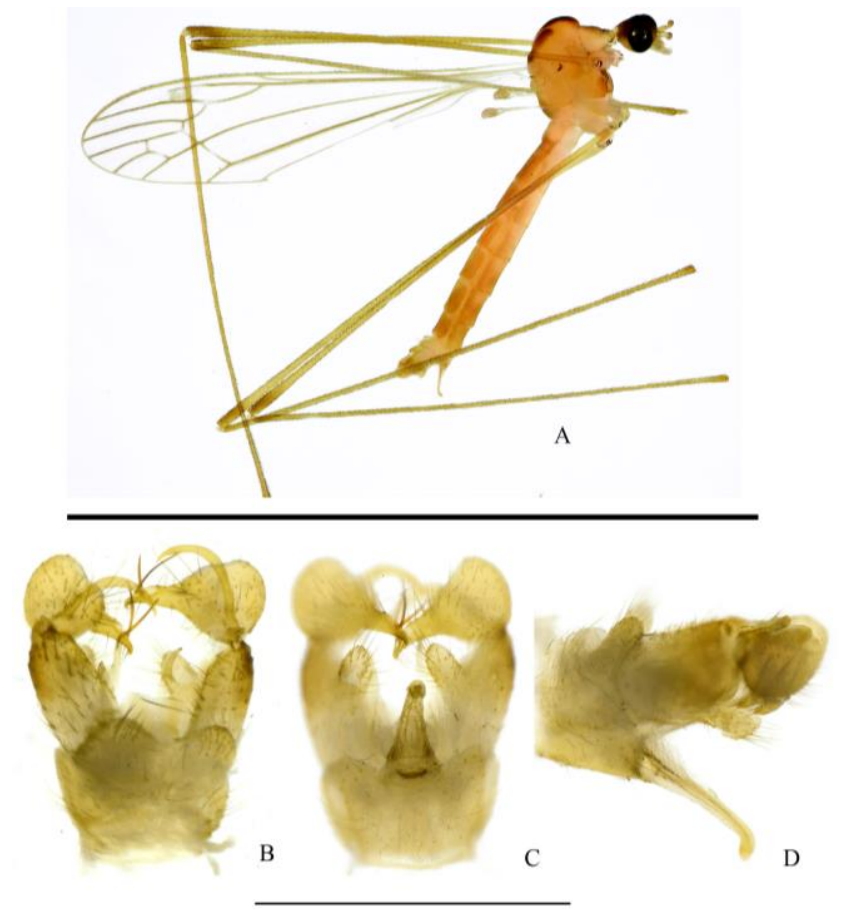

Figure 2. Dicranomyia (Dicranomyia) omissinervis de Meijere, 1918: A: male lateral habitus; B-D: male hypopygium B: dorsal view, C: ventral view, D: lateral view. Scale bars: A: $5 \mathrm{~mm}$; B-D: $1 \mathrm{~mm}$. 


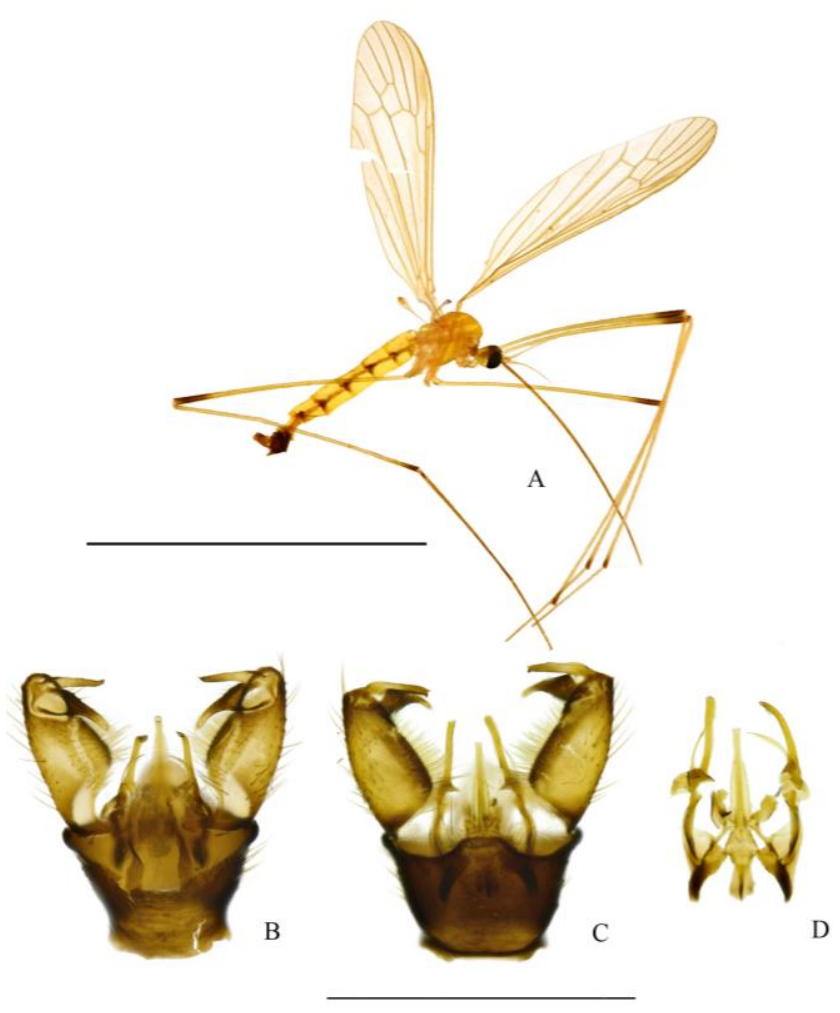

Figure 3. Elephantomyia (Elephantomyia) krivosheinae Savchenko, 1976: A: male lateral habitus; B-C: male hypopygium, B: dorsal view, C: ventral view, D: aedeagal complex in dorsal view. Scale bars: A: $10 \mathrm{~mm}$; B-D: $1 \mathrm{~mm}$

*Dicranomyia (Glochina) schineriana (Alexander, 1964)

Material. Temnikov Distr., MSNR, quarter 431, 02.VI.2016, 19, (RA); Romodanovo Distr., Pushkino, 17.VIII.2017, 1ㅇ, (RA); Saransk, 31.VIII.2018, 1へ̂, (RA).

Distribution. Palearctic species known from several countries, including Austria, Czech Rep., Germany, Hungary, Italy (Trentino-Alto Adige), Lithuania, Norway, Romania, Slovakia, Sweden, Switzerland, Ukraine, Kazakhstan, Kyrgyzstan; Mongolia. Russia: RUC (Chuvash Rep.), RUE, North Caucasus, WS (Altay, Tyva, Khakasiya Rep.).

\section{*Dicranomyia (Glochina) tristis (Schummel, 1829)}

Material. Ichalki Distr., NPS, Lvovskoe forestry, post Obrezki, 28-31.VIII.2017, 1ㅅ, 37우, (SG); $11-$ 14.IX.2017, 3 우, (SG); 18-20.IX.2017, 1ㅊ, 9우, (SG); Temnikov Distr., MSNR, quarter 442, 03.VI.2017, 1의, (RA); MSNR, quarter 86, 1ㅇ, (RA); MSNR, Pushta, at light, 02.VI.2014, 10َ, (RA).

Distribution. Widely distributed throughout the Palearctic Region; Russia: RUW, RUC (Tver and Yaroslavl regions), North Caucasus, WS (south), ES
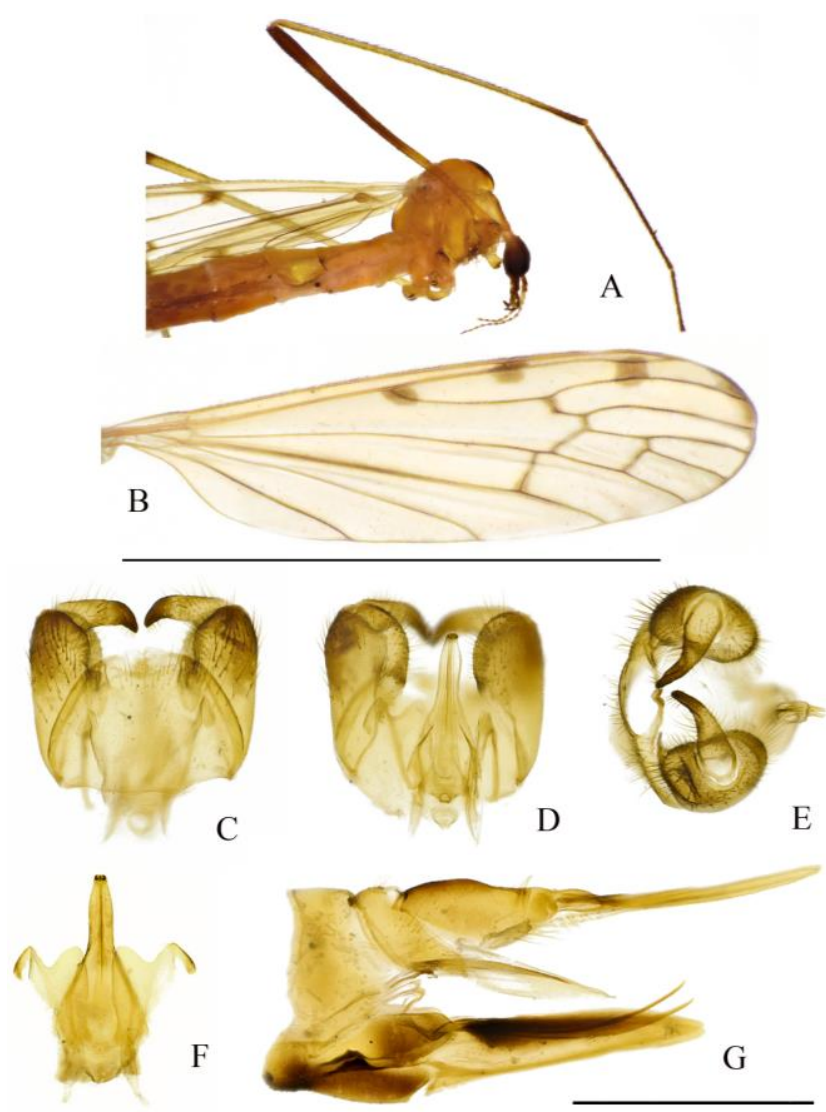

Figure 4. Limonia nigropunctata nigropunctata (Schummel, 1829): A: female lateral habitus; B: wing; C-E: male hypopygium, C: dorsal view, D: ventral view, E: caudal view; F: aedeagal complex in dorsal view; G: female ovipositor. Scale bars: A-B: 10 $\mathrm{mm}$; C-G: $1 \mathrm{~mm}$

(south), FE (south) (south Siberia eastward to Primorskiy Kray).

*Dicranomyia (Idiopyga) danica Kuntze, 1919

Material. Temnikov Distr., MSNR, Pushta, quarter 446, at light, 11.IX.2018, 1ڤึ, (SG).

Distribution. Known from several European countries, including Austria, Czech Rep., Denmark (incl. Faroe Is.), Finland (south), Germany, Great Britain, Hungary, Ireland, Lithuania, Netherlands, Poland, Slovakia, and Sweden. Also Turkey (Asiatic part), Kazakhstan (Pavlodar region), Kyrgyzstan; Russia: RUW (Pskov oblast), RUC (Tver region).

*Dicranoptycha fuscescens (Schummel, 1829)

Material. Temnikov Distr., MSNR, Pushta, quarter 446, at light, 11.IX.2018, 1ठ, (SG).

Distribution. Widely distributed in Europe, also in Morocco (Rif), Algeria, Georgia, Armenia, Azerbaijan, Turkey (Asiatic part), Cyprus, Lebanon, Israel, Iran, (?) Kazakhstan and Mongolia; Russia: RUW, RUC (Chuvash Rep.), RUE (Bashkortostan Rep., Tatarstan Rep.), North Caucasus. 
*Discobola parvispinula (Alexander, 1947)

Material. Ichalki Distr., NPS, Kemlyanskoe forestry, quarter 92, 11.VIII.2018, 10ิ, (RA); NPS, sanatorium Alatyr, at light, 16-17.VIII.2018, 2우, (SG); NPS, Lvovskoe forestry, post Obrezki, 11-14.IX.2017, 1, (SG); 18-20.IX.2017, 19, (SG).

Distribution. Known from several European countries, including Czech Rep., Lithuania, Poland, Slovakia, Ukraine (Carpathians). Widely distributed throughout the Palearctic Region; Russia: RUN (Karelia), RUC (Chuvash Rep.), RUE (Bashkortostan Rep., Kirovsk region), WS (Altay), ES (Krasnoyarskiy Kray), FE (Amur region, Primorskiy Kray, Sakhalin, Kuril Is.).

*Elephantomyia (Elephantomyia) krivosheinae Savchenko, 1976 (Figure 3)

Material. Temnikov Distr., MSNR, Pushta, at light, 22.V.2014, 1ð̄, (RA).

Distribution. Palearctic species are known from several European countries, including Czech Rep., Finland, Hungary, Lithuania, Poland, Slovakia, Sweden, Switzerland, Ukraine (west). Russia: RUN, RUC (Tver region), RUE, WS (Altay, Tyva), FE (Khabarovskiy Kray, Primorskiy Kray).

Remarks. The male genitalia are rarely illustrated and here we present the first photos of them (Figure 3).

*Helius (Helius) longirostris longirostris (Meigen, 1818)

Material. Ichalki Distr., NPS, sanatorium Alatyr, at

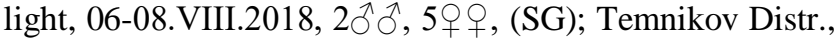
MSNR, quarter 421, 13.VI.2016, 19, (RA); MSNR, quarter 431, 02.VI.2016, 1q, (RA); Temnikov Distr., MSNR, post Steklyannyi, at light, 12-13.IX.2018, 1ठ̄, (SG).

Distribution. Widely distributed in Europe, also in Turkey (Asiatic part), Egypt and Israel; Russia: RUN (Arkhangelsk region), RUW, RUC (Tver and Yaroslavl regions), RUE, North Caucasus, WS (Altay).

\section{Limonia albifrons (Meigen, 1818)}

Literature. Ruchin and Pilipenko 2015.

Distribution. Known from several European countries, including Austria, Croatia, Czech Rep., France, Germany, Hungary, Italy (Trentino-Alto Adige), Lithuania, Montenegro, Poland, Romania, Serbia, Slovakia, Slovenia, Switzerland, Turkey (European part: Kirklareli), Ukraine; Russia: RUC (Rep. of Mordovia), WS (Altay).

*Limonia macrostigma (Schummel, 1829)

Material. Temnikov Distr., MSNR, Pushta, quarter 446, at light, 14-15.VIII.2018, 1, (SG).

Distribution. Widely distributed throughout the Palearctic Region; Russia: RUN, RUW, RUE, North Caucasus, WS (Altay), FE (Primorskiy Kray).

Remarks. This species is reported for the first time from Central European Russia and Mordovia.
*Limonia nigropunctata nigropunctata (Schummel, 1829) (Figure 4)

Material. Atyurievo Distr., Russian Velasma, 24.V.2014, 1ㅇ, (RA); Stepanovka, 24.V.2014, 1우, (RA); Temnikov Distr., Tretyakovo, 17.V.2014, 1q, (RA);

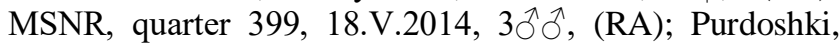
$19,(\mathrm{RA})$.

Distribution. Widely distributed in Europe, also in Turkey (Asiatic part); Russia: RUW, RUC (Chuvash Rep.). Remarks. The male and female genitalia are rarely illustrated and here we present the first photos of them.

\section{Limonia phragmitidis (Schrank, 1781)}

Literature. Ruchin and Pilipenko 2015.

Material. Elniki Distr., Malye Mordovskie Poshaty, 31.V.2014, 1ð̄, (RA); Staroe Shaygovo Distr., Nikolskaya Salovka, 08.VII.2017, 1ð̄, (RA); Temnikov Distr., MSNR, quarter 421, 13.VI.2016, 19, (RA); MSNR, quarter 435, 13.VI.2016, 2 우, (RA); 14.VI.2018, 1今, (RA); MSNR, Pushta, at light, 1§̂, (RA); MSNR, post Novenkovskiy, 13.VII.2014, 1 , , (RA).

Distribution. Widely distributed in Europe, also Morocco (Rif, Middle Atlas); Georgia, Azerbaijan, Turkey (Asiatic part), Israel, Jordan, Kazakhstan (Zailiyskiy Alatau Mts. S of Alma-Ata), Kyrgyzstan; Russia: RUN, RUW, RUC (Tver and Yaroslavl regions, Chuvash Rep.), RUE, North Caucasus, WS (Altay).

Limonia trivittata (Schummel, 1829)

Literature. Ruchin and Pilipenko 2015.

Distribution. Widely distributed throughout the Palearctic Region; Russia: RUN, RUW, RUC (Rep. of Mordovia, Tver region), RUE (Bashkortostan Rep.), North Caucasus, WS (Altay, Tyva), ?FE (?Kamchatka).

Metalimnobia (Metalimnobia) bifasciata (Schrank, 1781)

Literature. Ruchin and Pilipenko 2015.

Material. Bolshie Berezniki Distr., Nerley, 27.VIII.2016, 1ô, (RA); Insar Distr., Insar, 10.VI.2018, 10ิ, (RA); Ichalki Distr., NPS, Aleksandrovskoe forestry, quarter 39, 06.VI.2018, 1, (RA); NPS, Kemlyanskoe forestry, quarter 92, 11.VIII.2018, 12 $\hat{\jmath}, 6$ 우우, (RA); NPS, Lvovskoe forestry, quarter 63, 20.VII.2018, 3§え, 2 우우, (SG); NPS, Lvovskoe forestry, post Obrezki, 2831.VIII.2017, $2 \hat{\jmath}$, 3 우, (SG); 11-14.IX.2017, 1, (SG); Ichalki Distr., NPS, sanatorium Alatyr, at light, 0608.VIII.2018, 2우, (SG); 16-17.VIII.2018, 3ðへे, (SG); Romodanovo Distr., Pushkino, 17.VIII.2017, 19, (RA); Ruzaevka Distr., Levzhenskiy, 10.VI.2018, 10̄, (RA); Temnikov Distr., MSNR, quarter 431, 02.VI.2016, $1 \sigma^{\hat{\sigma}}$, (RA); MSNR, quarter 434, 1ð̄, (RA); 22.VIII.2015, $1 \hat{\jmath}$, (RA); MSNR, Pushta, at light, 1ð, (RA).

Distribution. Widely distributed throughout the Palearctic Region; Russia: RUN, RUW, RUC (Moscow, Tver, and Ulyanovsk regions, Chuvash Rep., Rep. of Mordovia), RUE, North Caucasus, WS (north), ES (Siberia north and eastward to Yakutia), FE (Kamchatka, Amur region, Primorskiy Kray, Sakhalin, Kuril Is.). 
Metalimnobia (Metalimnobia) quadrimaculata (Linnaeus, 1760)

Literature. Ruchin and Pilipenko 2015.

Material. Bolshie Berezniki Distr., Prissurskiy, 31.VIII.2018, 1ठ, (RA); Insar Distr., Insar, 1ठ,1우, (RA); Vasina Polyana, 10.VI.2018, 1ठ, (RA); Ichalki Distr., NPS, Aleksandrovskoe forestry, quarter 39, 06.VI.2018, $1 \delta, 1$, , (RA); quarter 31, 18.VI.2018, 1ㅇ, (RA); Ichalki Distr., NPS, Barakhmanovskoe forestry, quarter 113, 27.VIII.2018, 1§,, (RA); NPS, Kemlyanskoe forestry,

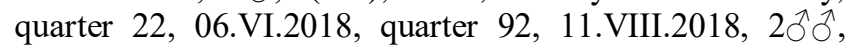
(RA); NPS, Lvovskoe forestry, quarter 63, 20.VII.2018,

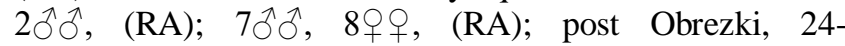
30.V.2018, 1ठ, (SG); 11-14.IX.2017, 1ð, (SG); NPS, sanatorium Alatyr, at light, 06-08.VIII.2018, 19, (SG); 1617.VIII.2018, 3우, (SG); Ruzaevka Distr., Levzhenskiy, 10.VI.2018, 4우, (RA); Khovanshchina, 10.VI.2018, 1엉, (RA); Temnikov Distr., MSNR, quarter 422, 21.VI.2018, 1ठ, (RA); MSNR, quarter 424, 05.VI.2018, $2 \hat{\jmath} \hat{\jmath}, 3$ 우, (RA); MSNR, quarter 426, 14.VI.2018, 4ㅊํ, 3우, (RA);

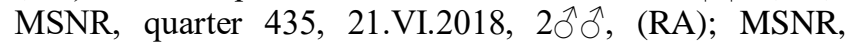
quarter 437, 26.VI.2018, 2 \%, , (RA); MSNR, quarter 439, 21.VI.2018, 3 + 9 , (RA); MSNR, quarter 440, 01.VI.2018, 2§ð, 3 웅, (RA); 14.VI.2018, 2 우, (RA); MSNR, post Inorskiy, 11-14.V.2018, 1§̂, 19, (RA); MSNR, Pushta, at light, 14.VIII.2017, 1§̂, (RA); MSNR, Srednyaya Melnitsa, soil trap, 29.VII-29.VIII.2018, 19, (SG).

Distribution. Widely distributed throughout the Palearctic Region; Russia: RUN, RUW, RUC (Moscow, Tver, Ulyanovsk, Yaroslavl regions, Chuvash Rep., Rep. of Mordovia), RUE (Bashkortostan Rep.), North Caucasus, WS (south), ES (south), FE (Kamchatka, Amur region, Primorskiy Kray, Sakhalin, Kuril Is.).

\section{Metalimnobia (Metalimnobia) quadrinotata (Meigen, 1818)}

Literature. Ruchin and Pilipenko 2015.

Material. Ichalki Distr., NPS, Lvovskoe forestry, post

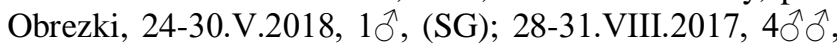
5 우, (SG); NPS, sanatorium Alatyr, at light, 16-

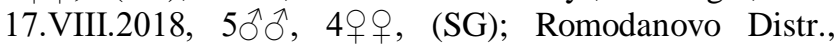
Pushkino, 17.VIII.2017, $2 \hat{\jmath} \widehat{\jmath}, 1$, , (RA); Temnikov Distr., MSNR, quarter 337, 11.VI.2016, 19, (RA); MSNR, quarter 347, 13.VII.2017, 19, (RA); MSNR, Drozhdenovkiy, 04.VI.2017, 1, (RA); MSNR, Pushta, at light, 22.V.2014, 1ठ, (RA).

Distribution. Widely distributed throughout the Palearctic Region; Russia: RUN, RUW, RUC (Moscow, Tver, Yaroslavl and Ulyanovsk regions, Chuvash Rep., Rep. of Mordovia), RUE, North Caucasus, WS, ES (east to Yakutia), FE (Magadan and Amur regions).

\section{Rhipidia (Rhipidia) maculata Meigen, 1818}

Literature. Ruchin and Pilipenko 2015.

Material. Ichalki Distr., NPS, Lvovskoe forestry, post Obrezki, 28-31.VIII.2017, 1ㅇ, (SG); 18-20.IX.2017, $1{ }_{0}$, 5우, (SG); NPS, sanatorium Alatyr, at light, 1617.VIII.2018, 3 우, (SG); Temnikov Distr., MSNR, post Steklyannyi, at light, 12-13.IX.2018, 1ð̄, 3우, (SG);
MSNR, Srednyaya Melnitsa, soil trap, 29.VII29.VIII.2018, 1우, (SG).

Distribution. The species is widespread in the Holarctic Region; Russia: RUN, RUW, RUC (Tver and Ulyanovsk regions, Rep. of Mordovia), RUE (Bashkortostan Rep.), North Caucasus, WS, ES, FE (from Magadan region and Kamchatka to Primorskiy Kray, Sakhalin and Kuril Is.).

\section{Family Pediciidae Osten Sacken 1860 \\ Subfamily Pediciinae Kertesz 1902}

* Dicranota (Paradicranota) gracilipes Wahlgren, 1905

Material. Ichalki Distr., NPS, Lvovskoe forestry, post Obrezki, 20-23.VIII.2018, 1q, (SG).

Distribution. Distributed in Europe; Russia: RUN, RUW, RUE (Bashkortostan Rep.).

Remarks. This species is reported for the first time from Central European Russia and Mordovia. There is also an unpublished record from Bashkortostan Republic (first record for the east European Russia, Gavryushin in litt., 2012).

\section{*Pedicia (Pedicia) rivosa rivosa (Linnaeus, 1758)}

Material. Ichalki Distr., NPS, Lvovskoe forestry, post Obrezki, 20-23.VIII.2018, 1ڤ̂, (SG).

Distribution. Distributed in Europe; Russia: RUN, RUW, RUC (Tver and Ulyanovsk regions, Chuvash Rep.), RUE (Bashkortostan Rep., Orenburg region), ?WS (?Altay).

\section{*Tricyphona (Tricyphona) immaculata (Meigen, 1804)}

Material. Insar Distr., Novley, 27.V.2017, 2へ̂ิ, (RA); Ichalki Distr., NPS, Lvovskoe forestry, post Obrezki, 2430.V.2018, 2ðึ, 6우, (SG); 18-20.IX.2017, 2 우, (SG); Temnikov Distr., MSNR, Pushta, at light, 13.V.2014, 3 우, (SG); MSNR, post Steklyannyi, at light, 12-13.IX.2018, $4 \hat{\jmath} \hat{\jmath}, 4$ 우, (SG).

Distribution. Widely distributed in Europe, also in Morocco (Rif), Georgia, Armenia, Azerbaijan, Turkey (Asiatic part), Lebanon and Kazakhstan (Zailiyskiy Alatau Mts. S of Alma-Ata), Kyrgyzstan; Russia: RUN, RUW, RUC (Tver region), RUE (Bashkortostan Rep.), North Caucasus, WS (Altay).

*Tricyphona (Tricyphona) unicolor (Schummel, 1829)

Material. Ichalki Distr., NPS, Lvovskoe forestry, post Obrezki, 24-30.V.2018, 3 우, (SG).

Distribution. Widely distributed in Europe, also in Turkey (Asiatic part); Russia: Novaya Zemlya, RUN, RUW, WS (Altay).

Remarks. This species is reported for the first time from Central European Russia and Mordovia.

\section{Subfamily Ulinae Alexander, 1966}

*Ula (Ula) bolitophila Loew, 1869

Material. Temnikov Distr., MSNR, Pushta, at light, 13.V.2014, 19, (RA); MSNR, Srednyaya Melnitsa, soil trap, 15.V.2018, 1, (SG). 
Distribution. Widely distributed throughout the Palearctic Region; Russia: RUN, RUW, RUE, WS (Altay), ES (south), FE (Khabarovskiy Kray, Primorskiy Kray, Sakhalin, Kuril Is.).

Remarks. This species is reported for the first time from Central European Russia and Mordovia.

\section{Family Tipulidae Linnaeus, 1758}

Subfamily Ctenophorinae Kertesz, 1902

Ctenophora (Cnemoncosis) fastuosa Loew, 1871

Literature. Ruchin and Pilipenko 2015.

Distribution. Known from several European countries, including Bulgaria, Croatia, Czech Rep., Germany, Poland (south), Romania, Ukraine (south); Also: Uzbekistan (south of Aral Sea); China (Heilongjiang, Zhejiang); Russia: RUC (Moscow region, Chuvash Rep., Rep. of Mordovia), RUS (north of Black Sea); WS (Tyva), FE (Amur region, Primorskiy Kray). A rare Palearctic species.

\section{Dictenidia bimaculata (Linnaeus, 1760)}

Literature. Ruchin and Pilipenko 2015.

Material. Temnikov Distr., MSNR, Pushta, at light, 08.VIII.2017, 1오, (RA).

Distribution. Widely distributed throughout the Palearctic Region; Russia: RUN, RUW, RUC (Moscow, Tver, Yaroslavl and Ulyanovsk regions, Chuvash Rep., Rep. of Mordovia), RUS, WS (southern half), ES, FE (Kamchatka, Sakhalin (incl. Moneron), Amur region, Primorskiy Kray).

\section{Tanyptera (Tanyptera) atrata atrata (Linnaeus, 1758)}

Literature. Ruchin and Pilipenko 2015.

Material. Bolshie Berezniki Distr., Nerley, 05.VI.2016, 1§, (RA); Temnikov Distr., Russkoe Karaevo, 21.V.2017, $1 \hat{\jmath}$, (RA); MSNR, quarter 360, 27.V.2016, 10े, (RA); MSNR, quarter 381, 28.V.2018, 10, (RA).

Distribution. Widely distributed throughout the Palearctic Region; Russia: RUN, RUW, RUC (Moscow, Nizhniy Novgorod, Tver, Yaroslavl, Ulyanovsk regions, Chuvash Rep., Rep. of Mordovia), RUS, WS (Altay), FE (Amur region).

\section{Subfamily Tipulinae Kertesz, 1902}

Nephrotoma aculeata (Loew, 1871)

Literature. Ruchin and Pilipenko 2015.

Material. Ichalki Distr., NPS, Lvovskoe forestry, post Obrezki, 20-23.VIII.2018, 1q, (SG); Krasnoslobodsk Distr., Staroe Sindrovo, 16.IX.2017, 1q, (RA); Staroe Shaygovo Distr., Staroe Shaygovo, 30.VII.2017, 19, (RA); Temnikov Distr., MSNR, Srednyaya Melnitsa, soil trap, 29.VII-29.VIII.2018, 2 \% , (SG); MSNR, Pushta, quarter 446, at light, 14-15.VIII.2018, 19, (SG); 28.VIII.2018, 1 , (SG); MSNR, post Steklyannyi, at light, 12-13.IX.2018, $1 \lesssim,(\mathrm{SG})$.

Distribution. Widely distributed throughout the Palearctic Region; Russia: RUN, RUW, RUC (Moscow, Yaroslavl regions, Chuvash Rep., Rep. of Mordovia), RUS, WS (south), ES (south), FE (south, incl. south Kamchatka, Sakhalin).
Nephrotoma analis (Schummel, 1833)

Literature. Ruchin and Pilipenko 2015.

Material. Temnikov Distr., MSNR, Pushta, at light, 18.VII.2014, 1今̄, (RA).

Distribution. Widely distributed throughout the Palearctic Region; Russia: RUN, RUW, RUC (Moscow, Tver and Yaroslavl regions, Chuvash Rep., Rep. of Mordovia), RUS, WS (southeast), ES (Minusinsk), FE (Amur region).

Nephrotoma appendiculata appendiculata (Pierre, 1919)

Literature. Ruchin and Pilipenko 2015.

Material. Temnikov Distr., MSNR, Plotomoyka, 16.VI.2018, 1 , , (RA).

Distribution. Widely distributed in Europe; Russia: RUN, RUW, RUC (Kaluga, Moscow, Tver, Yaroslavl, and Ulyanovsk regions, Chuvash Rep., Rep. of Mordovia), RUS.

Nephrotoma cornicina cornicina (Linnaeus, 1758)

Literature. Plavilshchikov 1964; Ruchin and Pilipenko 2015.

Material. Chamzinka Distr., Picheuri, 28.VII.2018, 2 우, (RA).

Distribution. The species is widespread and common in the Holarctic Region; Russia: RUN, RUW, RUC (Tula, Moscow, Tver, Yaroslavl and Ulyanovsk regions, Chuvash Rep., Rep. of Mordovia), RUS, WS (Ekaterinburg, south), ES (southern half), FE (south, incl. Sakhalin, Kuril Is.).

\section{Nephrotoma crocata (Linnaeus, 1758)}

Literature. Ruchin and Pilipenko 2015.

Distribution. Widely distributed in Europe; Russia: RUN, RUW, RUC (Tula, Nizhniy Novgorod, Moscow, Tver, Yaroslavl and Ulyanovsk regions, Chuvash Rep., Rep. of Mordovia), RUS, WS, ES

*Nephrotoma dorsalis (Fabricius, 1781)

Material. Temnikov Distr., MSNR, post Inorskiy, 25.VI.2014, 1ð̄, (RA).

Distribution. Widely distributed throughout the Palearctic Region; Russia: RUN, RUW, RUC (Moscow, Tver, and Yaroslavl regions, Chuvash Rep.), RUS (west), ES (south), FE (south, incl. Kamchatka).

*Nephrotoma lamellata lamellata (Riedel, 1910)

Material. Temnikov Distr., MSNR, quarter 409, 15.VI.2016, 1ठ, (RA); MSNR, quarter 360, 27.V.2016, $1 \hat{\jmath},(\mathrm{RA})$.

Distribution. Widely distributed throughout the Palearctic Region; Russia: RUW, RUC (Kaluga, Moscow regions, Chuvash Rep.,), WS (Altay, Tyva, Khakasiya Rep.), ES (south), FE (Amur region).

Nephrotoma pratensis pratensis (Linnaeus, 1758)

Literature. Ruchin and Pilipenko 2015.

Material. Ardatov Distr., Oktyabrskiy, 26.V.2017, 1ð, 1 ,,$(\mathrm{RA})$. 
Distribution. Widely distributed in Europe; Russia: RUN, RUW, RUC (Moscow, Tver, Yaroslavl and Ulyanovsk regions, Rep. of Mordovia), RUS, WS (southwest Chelyabinsk Territory).

*Nephrotoma quadristriata (Schummel, 1833)

Material. Ichalki Distr., NPS, Barakhmanovskoe forestry, quarter 101, 12.VIII.2018, 1, (RA).

Distribution. Widely distributed in Europe; Russia: RUN, RUW, RUC (Moscow region, Chuvash Rep.), RUS (west), WS (southeast), ES (southwest).

*Nephrotoma scalaris (Meigen, 1818)

Material. Temnikov Distr., MSNR, post Podrubnyi, 25.V.2014, 19, (RA); MSNR, Pushta, at light, 02.VI.2014, 1 ㅇ, (RA); MSNR, quarter 430, 11.VI.2016, 1ㅇ, (RA); MSNR, quarter 59, 23.VIII.2016, 1ठ, (RA).

Distribution. Widely distributed in Europe; Russia: RUC (Moscow, Tver and Yaroslavl regions, Chuvash Rep.), RUS.

\section{Nephrotoma scurra (Meigen, 1818)}

Literature. Plavilshchikov 1964; Antsiferova and Dobrosmyslov 1966; Ruchin and Pilipenko 2015.

Material. Bolshie Berezniki Distr., Simkinskoe lesnichestvo, 12.VIII.2017, 1q, (RA); Ichalki Distr., NPS, Barakhmanovskoe forestry, quarter 112, 12.VIII.2018, 1 , (RA); Lvovskoe forestry, quarter 70, 07.VI.2018, 1 Q (RA); NPS, Lvovskoe forestry, post Obrezki, 2831.VIII.2017, 1 , (SG); NPS, sanatorium Alatyr, at light, 06-08.VIII.2018, 2 우, (SG); 16-17.VIII.2018, 2 우, (SG); Temnikov Distr., MSNR, quarter 276, 04.VII.2017, 10̄, (RA); MSNR, quarter 319, 13.VII.2017, 10, (RA); MSNR, quarter 331, 09.VI.2016, 10, (RA); MSNR, quarter 384, 27.VII.2014, 2 우, (RA); MSNR, quarter 384, 14.VI.2016, $1 \hat{\jmath}$, (RA); MSNR, quarter 429, 15.VI.2016, 1 \%, (RA); MSNR, Pushta, quarter 446, at light, 14-15.VIII.2018, 19, (SG); 28.VIII.2018, 2우, (SG); Zhegalovo, 25.VII.2018, $1{ }^{\lambda},(\mathrm{SG})$.

Distribution. Widely distributed throughout the Palearctic Region; Russia: RUN, RUC (Voronezh, Ryazan, Moscow, Tver and Yaroslavl regions, Chuvash Rep., Rep. of Mordovia), RUS (west), WS, ES, FE.

*Nephrotoma tenuipes (Riedel, 1910)

Material. Ichalki Distr., NPS, sanatorium Alatyr, at light, 06-08.VIII.2018, 1ठ, (SG).

Distribution. Widely distributed throughout the Palearctic Region; Russia: RUW, RUC (Moscow, Tver, and Yaroslavl regions, Chuvash Rep.), North Caucasus, WS (Altay, Tyva), FE (Kamchatka).

*Nigrotipula nigra nigra (Linnaeus, 1758)

Material. Bolshie Berezniki Distr., 6 km SE Permisi, 12.VI.2015, 10َ, (RA).

Distribution. Widely distributed throughout the Palearctic Region; Russia: RUN, RUW, RUC (Voronezh, Moscow, and Tver regions, Chuvash Rep.), RUS (in Russia widespread between $63^{\circ} \mathrm{N}$ and $44^{\circ} \mathrm{N}$ ), WS, ES, FE (Amur region).
Prionocera subserricornis (Zetterstedt, 1851)

Literature. Ruchin and Pilipenko 2015.

Material. Temnikov Distr., MSNR, quarter 408, 10.V.2014, 19, (RA); MSNR, post Inorskiy, 11-14.V.2018, 1今, (SG); MSNR, post Podrubnyi, 15.V.2016, 4웅, (RA); MSNR, Pushta, at light, 25.VI.2014, 1, (RA); MSNR, Srednyaya Melnitsa, soil trap, 15.V.2018, 1ㅇ, (SG).

Distribution. The species is widespread in the Holarctic Region; Russia: RUN, RUW, RUC (Moscow, Tver, and Yaroslavl regions, Rep. of Mordovia), WS, ES, FE (Kamchatka, Sakhalin, and Kuril Is.).

Prionocera turcica (Fabricius, 1787)

Literature. Ruchin and Pilipenko 2015.

Distribution. The species is widespread in the Holarctic Region; Russia: RUN, RUW, RUC (Moscow, Yaroslavl, and Voronezh regions, Rep. of Mordovia), RUS, WS, ES, FE.

Tipula (Acutipula) fulvipennis De Geer, 1776

Literature. Ruchin and Pilipenko 2015.

Distribution. Widely distributed in Europe, also in Kazakhstan and Mongolia; Russia: RUN, RUW, RUC (Moscow, Ulyanovsk regions, Rep. of Mordovia), RUS, WS (south), ES (south).

*Tipula (Acutipula) luna Westhoff, 1879

Material. Temnikov Distr., MSNR, quarter 368, 27.V.2018, 3ðึ̄, 1ㅇ, (RA).

Distribution. Widely distributed in Europe; Russia: RUN, RUW, RUC (Moscow and Tver regions).

*Tipula (Lunatipula) affinis Schummel, 1833

Material. Tengushevo Distr., Barashevo, 07.VI.2014, $1 \hat{\jmath},(\mathrm{RA})$.

Distribution. Widely distributed in Europe, also Georgia, Armenia, Azerbaijan, Kazakhstan; Russia: RUN, RUW, RUC (Moscow, Tver, and Ulyanovsk regions), WS.

Tipula (Lunatipula) fascipennis Meigen, 1818

Literature. Ruchin and Pilipenko 2015.

Material. Ichalki Distr., NPS, Barakhmanovskoe forestry, quarter 98, 25.VII.2017, 1 9 , (RA); Kochkurovo Distr., Podlesnaya Tavla, 08.VII.2017, 19, (RA); Krasnoslobodsk Distr., Staraya Avgura, 01.VII.2018, 10̄, (RA); Temnikov Distr., MSNR, quarter 384, 17.VI.2014, 1 $\hat{0}$, (RA); MSNR, quarter 403, 5.VII.2015, 1q, (RA); MSNR, quarter 430, 30.VI.2015, 19 , (RA); MSNR, post Inorskiy, 28.VI.2015, 19, (RA); 02.VII.2017, 1Ô, (RA); MSNR, Pushta, at light, 03.VII.2014, 1, (RA).

Distribution. Widely distributed in Europe, also in Georgia, Armenia, Azerbaijan; Russia: RUN, RUW, RUC (Moscow, Tver, and Yaroslavl regions, Chuvash Rep., Rep. of Mordovia), RUS.

Tipula (Lunatipula) lunata Linnaeus, 1758

Literature. Ruchin et al 2007; Ruchin 2008; Ruchin and Pilipenko 2015.

Material. Bolshie Berezniki Distr., Permisi, 05.VI.2016, 19, (RA); Lyambir Distr., Novaya Uda, 
29.VII.2016, 1q, (RA); Staroe Shaygovo Distr., Nikolskaya Salovka, 08.VII.2017, 19, (RA); Temnikov Distr., MSNR, quarter 397, 17.VIII.2014, 1, (RA); MSNR, quarter 430, 11.VI.2016, 10̄, (RA); MSNR, Pushta, at light, 02.VI.2014, 1ठ, 3우, (RA); Purdoshki, 31.V.2014, 1ð,, (RA).

Distribution. Widely distributed throughout the Palearctic Region; Russia: RUN, RUW, RUC (Moscow, Tver, Yaroslavl and Ulyanovsk regions, Chuvash Rep., Rep. of Mordovia), RUS, WS, ES, FE.

\section{*Tipula (Lunatipula) mellea Schummel, 1833}

Material. Saransk, 1ð̄, (RA).

Distribution. Known from several European countries, including Austria, Belarus, Bulgaria, Croatia, Czech Rep., France, Germany, Hungary, Poland, Romania, Serbia, Slovakia, Ukraine also in Kazakhstan (east); Russia: RUC (Ulyanovsk, Voronezh regions), RUS.

\section{Tipula (Lunatipula) recticornis Schummel, 1833}

Literature. Ruchin and Pilipenko 2015.

Distribution. Known from several European countries, including Austria, Czech Rep., Finland (south), Germany, Lithuania, Poland, Romania, Slovakia, Sweden; also from Mongolia; Russia: RUC (Moscow region, Rep. of Mordovia), WS (north, Altay, Tyva), ES (north).

Tipula (Lunatipula) vernalis Meigen, 1804 2015.

Literature. Plavilshchikov 1964; Ruchin and Pilipenko

Material. Atyurievo Distr., Russian Velasma, 24.V.2014, 1ð̄, (RA); Bolshie Berezniki Distr., 6 km SE Permisi, 12.VI.2015, Bolshie Berezniki Distr., Nerley, 05.VI.2016, 05.VI.2016, 3 우, (RA); Permisi, 12.VI.2015, 1ㅇ, (RA); 05.VI.2016, 1ㅅ, 1, (RA); Dubensky Distr.,

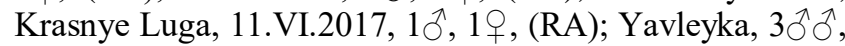
1, (RA); Elniki Distr., Malye Mordovskie Poshaty, 31.V.2014, 1§̂, 2qo, (RA); Insar Distr., Kochetovka, 04.VI.2016, 1ㅇ, (RA); NPS, Kemlyanskoe forestry, quarter 106, 08.VI.2018, 3 우, (RA); quarter 92, 07.VI.2018, 1ठ, 2우, (RA); quarter 94, 2 우, (RA); Kadoshkinsky Distr., Latyshovka, 04.VI.2016, 1ठ, 1ㅇ, (RA); Kovylkino Distr., Chepurnovka, 29.VI.2017, 19, (RA); Lyambir Distr., Atemar, 24.VI.2017, 1ð̄, 19, (RA); Oktyabrskiy Distr., Monastyrskoe, 12.VI.2017, 1へ,, (RA); Ruzaevka Distr.,

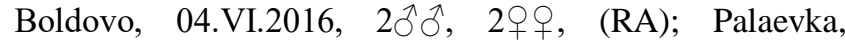
10.VI.2017, $3 \hat{\jmath} \widehat{\jmath}, 1$, (RA); Khovanshchina, 10.VI.2017, 1§̄, 3우, (RA); Temnikov Distr., MSNR, 22.V.2018,

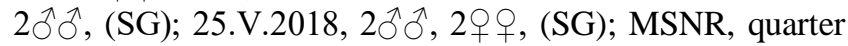
197, 18.VI.2016, 1q, (RA); MSNR, quarter 331, $1 \hat{O}^{\top}$, (RA); MSNR, quarter 368, 1今̄, (RA); MSNR, quarter 421, 08.VI.2014, 13.VI.2016, 1， (RA); 1ठ, 3우, (RA); MSNR, quarter 433, 03.VI.2016, 1q, (RA); MSNR, quarter 442, 02.VI.2016, 1§̄, (RA); 03.VI.2017, 1 ㅇ, (RA); MSNR, Drozhdenovkiy, 18.VI.2017, 1§, (RA); Alkaevo, 30.V.2015, 1ð̄, (RA); Purdoshki, 31.V.2014, 1q, (RA); Dachniy, 07.VI.2014, 1q, (RA); Torbeevo Distr., Surgod, 21.VI.2016, 1, (RA).

Distribution. Widely distributed in Europe; Russia: RUN, RUW, RUC (Smolensk, Moscow, Tver, Yaroslavl, and Ulyanovsk regions, Chuvash Rep., Rep. of Mordovia), North Caucasus.

*Tipula (Platytipula) autumnalis Loew, 1864

Material. Ichalki Distr., NPS, Lvovskoe forestry, post Obrezki, 11-14.IX.2017, 1ð̄, (SG).

Distribution. Known from several European countries, including Belarus, Czech Rep., Denmark, Estonia, Germany, Latvia, Lithuania, Netherlands, Poland, Sweden (Oland), Ukraine; Russia: RUN, RUW, RUC (Moscow, Tver, Yaroslavl, and Voronezh regions).

Tipula (Platytipula) luteipennis luteipennis Meigen, 1830

Literature. Ruchin and Pilipenko 2015.

Material. Ichalki Distr., NPS, Lvovskoe forestry, post Obrezki, 11-14.IX.2017, 15ðð, 10ㅇ, (SG); 18-

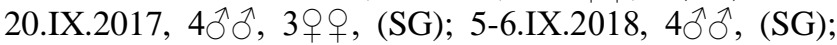
Temnikov Distr., MSNR, post Steklyannyi, at light, 12-

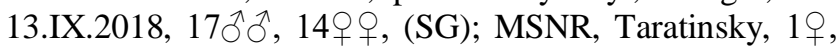
(RA); MSNR, Pushta, quarter 446, at light, 11.IX.2018, $16 \hat{\jmath} \hat{\jmath}, 5$ 오, $(\mathrm{SG})$.

Distribution. Widely distributed in Europe also in Kazakhstan (north, east), Kyrgyzstan, Mongolia; Russia: RUN, RUW, RUC (Moscow, Tver, Yaroslavl and Smolensk regions, Chuvash Rep., Rep. of Mordovia), RUS, WS (south), ES.

*Tipula (Pterelachisus) jutlandica Nielsen, 1947

Material. Temnikov Distr., MSNR, post Inorskiy, 1 , (RA).

Distribution. Known from several European countries, including Denmark, Finland (south), Germany, Lithuania, Sweden, and Ukraine; Russia: RUC (Moscow and Tver regions), WS (Altay).

*Tipula (Pterelachisus) laetibasis Alexander, 1934

Material. Temnikov Distr., MSNR, post Inorskiy, 1114.V.2018, 19, (SG); MSNR, post Steklyannyi, at light, 17.V.2018, $2 \overbrace{}^{\lambda} \sigma^{\lambda},(\mathrm{SG})$.

Distribution. Known from a small number of European countries, including Czech Rep., Finland, Norway (north), and Sweden and also from Japan (Honshu); Russia: RUN, RUC (Moscow region), WS (Altay), ES, FE (incl. Sakhalin, Kuril Is.).

Tipula (Pterelachisus) pseudovariipennis Czizek, 1912

Literature. Ruchin and Pilipenko 2015.

Distribution. Widely distributed in Europe; Russia: RUW, RUC (Moscow region, Chuvash Rep., Rep. of Mordovia) RUS.

\section{Tipula (Pterelachisus) submarmorata Schummel, 1833}

Literature. Ruchin and Pilipenko 2015.

Material. Ichalki Distr., NPS, Lvovskoe forestry, post

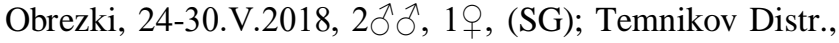
MSNR, quarter 342, 25.V.2015, 1§̄, (RA); MSNR, quarter 442, 03.VI.2017, 1 9 , (RA); MSNR, post Steklyannyi, at light, 17.V.2018, 1今, 2ᄋㅇ, (SG); MSNR, Srednyaya Melnitsa, soil trap, 15.V.2018, 1ठ̄, (SG). 
Distribution. Widely distributed in Europe; Russia: RUN, RUW, RUC (Moscow and Tver regions, Rep. of Mordovia).

Tipula (Pterelachisus) varipennis Meigen, 1818

Literature. Ruchin and Pilipenko 2015.

Material. Ichalki Distr., NPS, Lvovskoe forestry, post Obrezki, 24-30.V.2018, 1ð̄, 2우, (SG); Temnikov Distr., Tretyakovo, 17.V.2014, 1ठ, 1 오 (RA).

Distribution. Widely distributed in Europe, Siberia and in Kazakhstan (east); Russia: RUN, RUW, RUC (Moscow, Tver, and Yaroslavl regions, Rep. of Mordovia), RUS, WS (south), ES (Krasnoyarskiy Kray, Irkutsk region).

*Tipula (Pterelachisus) winthemi Lackschewitz, 1932

Material. Temnikov Distr., MSNR, Srednyaya Melnitsa, soil trap, 15.V.2018, 1ð̄, 1, (SG); Zubova Polyana Distr., Yavas, 22.VI.2018, 19 (RA).

Distribution. Known from several European countries, including Austria, Belgium, Croatia, Denmark, Estonia, Finland, Germany, Lithuania, Netherlands, Spain (north), Sweden (north), Switzerland, and Ukraine; also from Mongolia; Russia: RUC (Moscow region), WS (Ob delta), ES (Irkutsk region), FE (Kamchatka).

Tipula (Savtshenkia) obsoleta Meigen, 1818

Literature. Ruchin and Pilipenko 2015.

Material. Ichalki Distr., NPS, Lvovskoe forestry, post

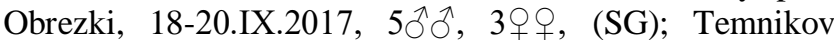
Distr., MSNR, post Steklyannyi, at light, 12-13.IX.2018, $2 \hat{\jmath},(\mathrm{SG})$.

Distribution. Widely distributed in Europe, also in Georgia, Turkey (Asiatic part: Trabzon), Kazakhstan (east); Russia: RUN, RUW, RUC (Moscow, Tver, Yaroslavl, Smolensk regions, Chuvash Rep., Rep. of Mordovia).

Tipula (Tipula) paludosa Meigen, 1830

Literature. Plavilshchikov 1964; Timraleev 1992; Ruchin et al 2007; Ruchin 2008; Ruchin and Pilipenko 2015.

Material. Atyurievo Distr., Mordovskaya Kozlovka, 10.VIII.2014, 1ठ, (RA); Ichalki Distr., NPS, Lvovskoe forestry, post Obrezki, 20-23.VIII.2018, 4ðึ, (SG); Temnikov Distr., MSNR, quarter 324, 16.VIII.2015, 1 ㅇ (RA); MSNR, quarter 399, 17.VIII.2014, 1ڤ̂, (RA); MSNR, quarter 420, 06.VIII.2017, 1§, (RA); MSNR, quarter 85, 23.VIII.2016, 10े, (RA); MSNR, post Steklyannyi, at light, 12-13.IX.2018, 1ð̄, (SG); MSNR, Pushta, quarter 446, at light, 14-15.VIII.2018, 3ðे, (SG).

Distribution. Widely distributed in Europe and in North America; Russia: RUN, RUW, RUC (Moscow, Tver, Ulyanovsk, Yarovslavl, and Tula regions, Rep. of Mordovia), RUS.

Tipula (Tipula) subcunctans Alexander, 1921

Literature. Timraleev 1992; Pilipenko 2009; Ruchin and Pilipenko 2015.

Material. Ichalki Distr., NPS, Lvovskoe forestry, post Obrezki, 11-14.IX.2017, 11ठิð, 20우, (SG); 1820.IX.2017, 2ふึ่, 2우, (SG).
Distribution. Widely distributed throughout the Palearctic Region; Russia: RUN, RUW, RUC (Moscow, Tver, and Yaroslavl regions, Chuvash Rep., Rep. of Mordovia), RUS, WS (south), ES (south, Yakutia), FE (south, incl. Sakhalin).

Tipula (Vestiplex) hortorum Linnaeus, 1758

Literature. Ruchin and Pilipenko 2015.

Material. Temnikov Distr., MSNR, quarter 403, 20.V.2017, 1今, (RA); MSNR, quarter 408, 20.V.2017, $1 \hat{\jmath}$, (RA); MSNR, quarter 448, 11.V.2016, $1 \hat{\jmath}$, (RA); MSNR, Pushta, at light, 13.V.2014, 1 9 (RA).

Distribution. Widely distributed in Europe; Russia: RUN, RUW, RUC (Moscow, Tver, Nizhniy Novgorod and Ulyanovsk regions, Chuvash Rep., Rep. of Mordovia), RUS.

Tipula (Vestiplex) nubeculosa Meigen, 1804

Literature. Ruchin and Pilipenko 2015.

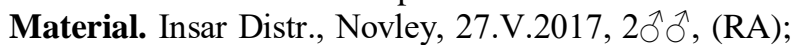
Temnikov Distr., MSNR, quarter 338, 27.V.2018, 1옹, (RA); MSNR, quarter 408, 10.V.2014, 10 , (RA); MSNR, quarter 448, 24.V.2015, 1ठ, (RA); MSNR, post Inorskiy,

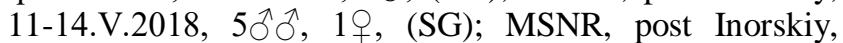
21.V.2017, 1q, (RA); MSNR, post Polyansky, 18.V.2014, 1§̂, (RA); MSNR, Pushta, at light, 12.V.2014, 1q, (RA); MSNR, Srednyaya Melnitsa, soil trap, 15.V.2018, 1 ${ }_{0}$, (SG).

Distribution. Widely distributed in Europe; Russia: RUN, RUW, RUC (Moscow, Tver, and Ulyanovsk regions, Chuvash Rep., Rep. of Mordovia), RUS.

Tipula (Vestiplex) scripta scripta Meigen, 1830

Literature. Ruchin and Pilipenko 2015.

Material. Ichalki Distr., NPS, sanatorium Alatyr, at light, 06-08.VIII.2018, 3ㅅ, 30 ㅇ, (SG); NPS, Kemlyanskoe forestry, quarter 92, 11.VIII.2018, 1 , , (RA); NPS, Lvovskoe forestry, post Obrezki, 20-23.VIII.2018, 1 , , (SG).

Distribution. Widely distributed throughout the Palearctic Region; Russia: RUN, RUW, RUC (Moscow, Tver, Yaroslavl, Ulyanovsk, Nizhniy Novgorod and Voronezh regions, Chuvash Rep., Rep. of Mordovia), RUS, WS (south), ES (south), FE (Sakhalin).

Tipula (Yamatotipula) couckei Ton. 1921

Literature. Ruchin and Pilipenko 2015.

Distribution. Widely distributed throughout the Palearctic Region. Russia: RUN, RUW, RUC (Bryansk, Moscow, Tver, Ulyanovsk, and Voronezh regions, Rep. of Mordovia), RUS, WS (Altay, Tyva), ES (Krasnoyarskiy Kray).

Tipula (Yamatotipula) lateralis Meigen, 1804

Literature. Ruchin and Pilipenko 2015.

Material. Kochkurovo Distr., Novaya Pyrma, 11.VIII.2017, 1ठ઼, (RA); Temnikov Distr., MSNR, Pushta, quarter 446, at light, 29.IX.2018, 3우, (SG); MSNR, Srednyaya Melnitsa, soil trap, 29.VII-29.VIII.2018, 1ðึ, $19,(\mathrm{SG})$. 
Distribution. Widely distributed in Europe, also in Georgia, Armenia, Turkey (Asiatic part: widespread), Kazakhstan (east), Turkmenistan. Russia: RUN, RUW, RUC (Moscow, Tver, Yaroslavl, Ulyanovsk, and Voronezh regions, Rep. of Mordovia), RUS.

\section{*Tipula (Yamatotipula) pierrei Tonnoir, 1921}

Material. Ichalki Distr., NPS, Lvovskoe forestry, post Obrezki, 28-31.VIII.2017, 1q, (SG); 20-23.VIII.2018, $4 \widehat{\jmath}, 3 ㅇ+$, (SG); NPS, sanatorium Alatyr, at light, 0608.VIII.2018，19，(SG); 16-17.VIII.2018, 19, (SG); Temnikov Distr., MSNR, Pushta, quarter 446, at light, 19 , (SG).

Distribution. Widely distributed throughout the Palearctic Region. Russia: RUN, RUW, RUC (Moscow, Tver, Yaroslavl, Ulyanovsk, Smolensk and Ryazan regions), RUS, WS, ES, FE (incl. Kuril Is.).

\section{Conclusion}

As a result of this study, the known diversity of craneflies in the Republic of Mordovia significantly exceeds that of the neighboring regions. For example, the list of the most researched, Chuvash Republic includes 67 species (Paramonov 2016, 2017, 2018). However, the fauna of Mordovia has not yet been fully explored and requires further study.

\section{ACKNOWLEDGEMENTS}

We are grateful to Roman Rakitov (Paleontological Institute of Russian Academy of Sciences, Moscow, Russia) for checking and improving the English text.

\section{REFERENCES}

Antsiferova TA, Dobrosmyslov PA. 1966. Entomofauna of oats vetchphacelium and oats-vetch mixtures in the Mordovian ASSR. In: Ecological-faunistica relationships of selected groups of invertebrates and vertebrates. Mordovia Book Publishing House, Saransk. [Russian]

Astakhov DM, Ruchin AB, Romadina OD, Pristrem IM. 2019. To robber flies fauna (Diptera: Asilidae) of Mordovia, Russia. Biodiversitas 20 (4): 994-1005. DOI: 10.13057/biodiv/d200409.

Barkalov AV, Saaya AD. 2014. Crane flies (Diptera, Tipulidae) of Mountain Altai. Euroasian Entomol J 13: 59-68. [Russian with English abstract]

Budaeva IA, Ruchin AB. 2014. To the fauna of blackflies (Diptera: Simuliidae) of the Republic of Mordovia (Russia). Caucasian Entomol Bull 10 (1): 155-159. [Russian]

Champlain AB, Knull JN. 1932. Fermenting bait traps for trapping Elateridae and Cerambycidae (Coleop.). Entomol News 43 (10): 253 257.

Chursina MA, Ruchin AB. 2018a. A checklist of Syrphidae (Diptera) from Mordovia, Russia. Halteres 9: 57-73. DOI 10.5281/zenodo. 1255874

Chursina MA, Ruchin AB. 2018b. A checklist of Bombyliidae (Diptera) from Mordovia, Russia and variation of wing shape in Bombylius species. Biodiversitas 19: 2147-2156. DOI: 10.13057/biodiv/d190622

Egorov LV, Ivanov AV. 2018. Beetles (Insecta, Coleoptera), collected by fermenting bait crown traps in Chuvashia. Proceedings of the Mordovia State Nature Reserve 21: 191-204.

Humala AE, Polevoi AV. 2009. On the insect fauna of South-East Karelia Transactions of the Karelian Research Centre of the Russian Academy of Science) 4 (4): 53-75. [Russian with English summary]
Lantsov VI. 2009a. New for the Central Caucasus species of tipuloid dipterans (Diptera, Tipulidae, Limoniidae) in the autumn fauna of Rtzivashki valley (the North Caucasus). In: Fauna of mountain territories. KMK Scientific Press, Moscow. [Russian]

Lantsov VI. 2009b. New records for Limoniidae and Pediciidae (Diptera) from the Caucasus, Russia. In: Lantsov V (ed) Crane flies. History, taxonomy and ecology (Diptera: Tipulidae, Limoniidae, Pediciidae, Trichoceridae, Ptychopteridae, Tanyderidae). Memorial volume dedicated to Dr. Charles Paul Alexander (1889-1981), Dr. Bernhard Mannheims (1909-1971) and Dr. Evgeniy Nikolaevich Savchenko (1909-1994). Zoosymposia 3: 111-114.

Lantsov VI. 2009c. The ecology, biology and larval instars of the North Caucasian population (Lake Maliy Tambukan) of Tipula subcunctans Alexander, 1921 (Diptera: Tipulidae). In: Lantsov V (ed) Crane flies. History, taxonomy and ecology (Diptera: Tipulidae, Limoniidae, Pediciidae, Trichoceridae, Ptychopteridae, Tanyderidae). Memorial volume dedicated to Dr. Charles Paul Alexander (1889-1981), Dr. Bernhard Mannheims (1909-1971) and Dr. Evgeniy Nikolaevich Savchenko (1909-1994). Zoosymposia 3: 115-129.

Lantsov VI. 2011a. A paleoendemic limoniid species of the genus Ilisia (Diptera, Limoniidae) new for the fauna of Russia and the northern Caucasus [Russian with English summary]. Zoologicheskiy Zhurnal 90: 632-633 (English translation: Entomol Rev 91: 811-812 (2011)).

Lantsov VI. 2011b. Ecological groups of tipuloid dipteran larvae (Diptera: Tipuloidea) of the Caucasus. In: Problems of soil zoology. Materials of XVI All-Russia Conference on Soil Zoology. Moscow-Rostov: 6971

Lantsov VI. 2011c. Crane-flies (Diptera, Tipulidae) and limoniids (Limoniidae) of Dolgij island (Barents sea). In: International East European Symposium on Entomology. The Modern Problems of Entomology. Materials of the Conference. Voronezh State University, 18-21 November 2011: 83-88 [Russian]

Lantsov VI. 2012a. Ecology of the Caucasus populations and preimaginal spages of the crane-fly Tipula nigroantennata Sav. (Diptera, Tipulidae). Russian Entomol J 21: 269-272.

Lantsov VI. 2012b. New data on taxonomy, ecology and behaviour of craneflies of the genus Hexatoma Latr. (Diptera, Limoniidae) of the Caucasus. Materials of the XIV Congress of the Russian Entomological Society, Saint Petersburg, August 27 - September 1, 2012: 193 [Russian].

Lantsov VI. 2012c. New for the Central Caucasus: Dicranota parviuncinata Sav. (Diptera: Pediciidae), with data on its distribution and ecology. Caucasian Entomol Bull 8: 339-341.

Lantsov VI. 2014a. The tipuloid dipterans (Diptera, Tipulidae, Limoniidae) from the Putorana Plateau, with description of Dactylolabis tschernovi sp. n. [Russian with English summary]. Zoologicheskiy Zhurnal 93: 179-184 (English translation: Entomol Rev 94: 613-618 (2014)).

Lantsov VI. 2014b. The genus Prionocera (Diptera: Tipulidae) in the fauna of the Caucasus. Caucasian Entomol Bull 10: 151-153.

Lantsov VI. 2014c. Elephantomyia edwardsi Lackschewitz, 1932 (Diptera: Limoniidae) in the fauna of the Caucasus: distribution and ecology. Caucasian Entomol Bull 10: 283-285.

Lantsov VI. 2015a. New records of Limoniid-flies (Diptera: Limoniidae) from the North Caucasus. Proceedings of Samarskiy Scientific Center of the Russian Academy of Sciences 4: 365-369. [Russian]

Lantsov VI. 2015b. A new species of the genus Geranomyia Haliday, 1833 (Diptera: Limoniidae) from Transcaucasia. Caucasian Entomol Bull 11: 411-414.

Lantsov VI. 2016. The population of Tipuloid Dipterans (Diptera: Pediciidae, Limoniidae, Tipulidae) of wood belt communities of Arkhyz (the north-western Caucasus). Proceedings of X All-Russian Dipterological Symposium. Kuban State University. Krasnodar, 2328 August 2016: 165-170. [Russian]

Lantsov VI. 2017a. The crane flies (Diptera: Limoniidae) of Krasnodar Territory (the West Caucasus, Russia). Taxonomic diversity and ecology. XV Congress of the Russian Entomological Society. Russia. Novosibirsk, July 31-August 7, 2017. Materials of the Congress: 286287. [Russian]

Lantsov VI. 2017b. Some dominant species of the crane flies (Diptera, Tipuloidea) of the North Caucasus as biological indicators of habitats. In: Mountain ecosystems and their components. Materials of VI AllRussian conference with international participation. 11-16 September 2017, Nalchik: 106-107 [Russian]

Lantsov VI. 2017c. New records of crane flies (Diptera: Tipuloidea: Limoniidae, Tipulidae) in the spring fauna of Agrakhanski and 
Kizlyarski territories of Daghestan state nature reserve (the North Caucasus). Proceedings of Samarskiy Scientific Center of the Russian Academy of Sciences Vol. 19, No. 5 (2): 284-290. [Russian]

Lantsov VI. 2018. Habitats of the Northern Caucasus crane fly Tipula (Lunatipula) sublunata (Diptera: Tipulidae). In: Materials of XVIII All-Russia Conference on Soil Zoology. Moscow: 118-119. [Russian]

Lantsov VI. 2019. Data on crane flies (Diptera: Tipuloidea) of Dagestan (the North East of the Caucasus). In: Chamutiova T, Hamerlik L (eds) The 10th Central European Dipterological Conference, Kezmarske Zlaby, 23rd-25th September, 2019. Faculty of Natural Sciences, Matej Bel University, Banska Bystrica: Conference Abstracts 55-56.

Lantsov VI, Bibin AR. 2019. Ctenophora flaveolata (Fabricius, 1794) (Diptera: Tipulidae), a crane fly species new for Russia and the Caucasus. Caucasian Entomol Bull 15: 207-209.

McAlpine JF. 1981. Morphology and terminology, adults. In: McAlpine JF, Peterson BV, Shewell GE, Teskey HJ, Vockeroth JR, Wood DM (Coordinators), Manual of Nearctic Diptera. Biosystematic Research Centre, Ottawa, Ontario.

Makarkin VN, Ruchin AB. 2018. New data on Neuroptera and Raphidioptera of Mordovia (Russia). Caucasian Entomol Bull 15 (1) 147-157. [Russian].

Fasulati KK. 1971. Field studying of land invertebrates. Vol. 2. Vysshaya Shkola, Moscow. [Russian]

Krivosheina NP. 2009. Morphological characterization of the larvae and pupae of Epiphragma (Diptera, Limoniidae). Zoologicheskiy Zhurnal 88: 454-464. [Russian with English summary] (English translation: Entomol Rev 89: 340-350.

Krivosheina MG, Krivosheina NP. 2010. New data on morphology and ecology of limoniid fly larvae of the genus Metalimnobia (Diptera Limoniidae) developed in fungal substrates. Zoologicheskii Zhurnal 89: 1098-1117 [Russian with English summary] (English translation: Entomol Rev 90: 764-782 (2010).

Krivosheina NP, Krivosheina MG. 2019. Saproxylic Diptera (Insecta) of the Lazovsky State Nature Reserve (Russia). Nat Conserv Res 4 (3): 78-92. DOI: $10.24189 / \mathrm{ncr} .2019 .052$.

Oosterbroek P. 2019. Catalogue of the Craneflies of the world (Diptera, Tipuloidea, Pediciidae, Limoniidae, Cylindrotomidae, Tipulidae) www.ccw.naturalis.nl

Paramonov NM. 2006. To learn the fauna of tipuloid (Tipuloidea: Diptera) in Ulyanovsk Province. The Nature of Simbirsk Part of Volga Region 7: 148-153. [Russian]

Paramonov NM. 2011. To craneflies fauna (Diptera: Tipuloidea) of Mariy El Republic. Entomological and Parasitological Research in Volga Region, Saratov 9: 41-43. [Russian]

Paramonov NM. 2012. To craneflies fauna (Diptera: Tipuloidea) of Mariy El republic. Part II, National park Mariy Chodra. Entomological and Parasitological Research in Volga Region, Saratov 10: 110-111 [Russian]

Paramonov NM. 2014. Revision of the Tipuloidea (Diptera) from the collection by E.A. Eversmann. Eversmannia 39: 39-41 [Russian]

Paramonov NM, Klepikov MA. 2014. Annotated checklist of the Tipuloidea (Diptera) of the Yaroslavl Province. Eversmannia 39: 35 38 [Russian]

Paramonov NM. 2016. Annotated checklist of the Tipuloidea (Diptera) of the Chuvash Republic. Proceedings of the National Nature Reserve Prisursky 31: 130-132. [Russian]

Paramonov NM, Pilipenko VE. 2016. Annotated checklist of the Tipuloidea (Diptera) of the Tver province. Eversmannia 47-48: 96102. [Russian]

Paramonov NM. 2017. Annotated checklist of the Tipuloidea (Diptera) of the Chuvash Republic. Part II. Proceedings of the National Nature Reserve Prisursky 32: 171-174. [Russian]

Paramonov NM. 2018. Annotated checklist of the Tipuloidea (Diptera) of the Chuvash Republic. Part III. Proceedings of the National Nature Reserve Prisursky 33: 182-186. [Russian]

Pilipenko VE. 2008. A record of the Far Eastern crane fly Tipula (Platytipula) moiwana (Diptera, Tipulidae) in Moscow. Entomol Rev 88 (6): 747-747. DOI: 10.1134/S0013873808060146

Pilipenko VE. 2009. A checklist of craneflies (Diptera, Tipulidae) of the Central European territory of Russia. In: Lantsov V (ed) Crane flies. History, taxonomy and ecology (Diptera: Tipulidae, Limoniidae, Pediciidae, Trichoceridae, Ptychopteridae, Tanyderidae). Memoria volume dedicated to Dr. Charles Paul Alexander (1889-1981), Dr. Bernhard Mannheims (1909-1971) and Dr. Evgeniy Nikolaevich Savchenko (1909-1994). Zoosymposia 3: 203-220.
Plavilshchikov NN. 1964. A list of insect species found in the Mordovian State Nature Reserve. Proceedings of the Mordovia State Nature Reserve 2: 105-134. [Russian]

Polevoi AV, Salmela J. 2014. New data on the distribution of Limonia badia Walker and Gnophomyia acheron Alexander (Diptera, Limoniidae) in eastern Fennoscandia. Trudy Karelskogo Nauchnogo Tsentra RAN (Transactions of the Karelian Research Centre of the Russian Academy of Science) 2014 (2): 168-170.

Polevoi AV, Pilipenko VE. 2016. The first records of the crane flies Tipula apicispina and T. stenostyla (Diptera: Tipulidae) from Russian Karelia with new data on their bionomics. Zoosyst Rossica 25 (2): 380-386.

Polevoi AV, Ruokolainen A, Shorohva E, 2018. Eleven remarkable Diptera species, emerged from fallen aspens in Kivach Nature Reserve, Russian Karelia. Biodivers Data J 6 (e22175): 1-22.

Przhiboro AA. 2003. New records of craneflies from NW Russia, with ecological notes on some species (Diptera, Tipulidae, Limoniidae). Zoosyst Rossica 11: 361-366.

Przhiboro AA. 2009. Two new records of Tipuloidea (Diptera) from a cold spring in NW Russia. In: Lantsov V (ed) Crane flies. History, taxonomy and ecology (Diptera: Tipulidae, Limoniidae, Pediciidae, Trichoceridae, Ptychopteridae, Tanyderidae). Memorial volume dedicated to Dr. Charles Paul Alexander (1889-1981), Dr. Bernhard Mannheims (1909-1971) and Dr. Evgeniy Nikolaevich Savchenko (1909-1994). Zoosymposia 3: 229-233.

Przhiboro AA. 2017. New data on psammorheophilous Limoniidae (Diptera) of NE European Russia. Zoosyst Rossica 26: 381-391.

Ruchin AB. 2008. List of insect species of National Park «Smolny». Proceedings of the National Park 1: 151-180. [Russian]

Ruchin AB. 2018. Biology and distribution of the Clouded Apollo Parnassius mnemosyne (Linnaeus, 1758) (Lepidoptera: Papilionidae), a rare butterfly in the Republic of Mordovia, Russia. J Threatened Taxa 10 (7): 11980-11983.

Ruchin A, Antropov A. 2019. Wasp fauna (Hymenoptera: Bethylidae, Chrysididae, Dryinidae, Tiphiidae, Mutillidae, Scoliidae, Pompilidae, Vespidae, Sphecidae, Crabronidae \& Trigonalyidae) of Mordovia State Nature Reserve and its surroundings in Russia. J Threatened Taxa 11 (2): 13195-13250. DOI: 10.11609/jot.4216.11.2.1319513250 .

Ruchin AB, Artaev ON. 2016. On expansion of the distribution range of some scoliid wasps (Scoliidae, Hymenoptera, Insecta) in the Middle Volga region. Res J Pharm Biol Chem Sci 7 (3): 2110-2115.

Ruchin AB, Loginova NG, Kurmaeva DK. 2007. The entomofauna of two forestries of National Park. In: Fauna and ecology of insects, Vol. 1. Publisher «Tsentr valeologii vuzov Rossii», Rostovon-Don. [Russian]

Ruchin AB, Pilipenko VE. 2015. Preliminary checklist of the Tipuloidea (Diptera, Limoniidae, Tipulidae) of the Mordovia Republic. Eversmannia 41: 57-60. [Russian]

Ruchin AB, Makarkin NV. 2017. Neuroptera and Raphidioptera in the Mordovia State Nature Reserve. Nature Conservation Research 2 (2): 38-46. DOI: $10.24189 /$ ncr.2017.001. [Russian]

Ruchin AB, Egorov LV. 2017. Overview of insect species included in the Red Data Book of Russian Federation in the Mordovia State Nature Reserve. Nat Conserv Res 2 (Suppl. 1): 2-9. DOI: 10.24189/ncr.2017.016. [Russian]

Ruchin AB, Egorov LV. 2018a. Fauna of longicorn beetles (Coleoptera: Cerambycidae) of Mordovia. Russian Entomol J 27: 161-177. DOI: 10.15298/rusentj.27.2.07

Ruchin AB, Egorov LV. 2018b. Leptura aurulenta (Coleoptera, Cerambycidae), a new record of a very rare species in Russia. Nat Conserv Res 3 (1): 88-91. DOI:10.24189/ncr.2018.003

Ruchin AB, Egorov LV, Semishin GB. 2018. Fauna of click beetles (Coleoptera: Elateridae) in the interfluve of Rivers Moksha and Sura, Republic of Mordovia, Russia. Biodiversitas 19: 1352-1365. DOI: 10.13057/biodiv/d190423

Ruchin AB, Grishutkin GF. 2018. Biology and distribution of Parnassius apollo (Linnaeus, 1758) a rare species in Mordovia Republic, Russia. Biodiversitas 19 (2): 431-436. DOI: 10.13057/biodiv/d190210

Ruchin AB, Mikhailenko AP. 2018. Fauna of mantids and orthopterans (Insecta: Mantodea, Orthoptera) of the Mordovia State Nature Reserve, Russia. Biodiversitas 19: 1194-1206. DOI: 10.13057/biodiv/d190403 Ruchin

Ruchin AB, Alekseev SK, Khapugin AA. 2019a. Post-fire fauna of carabid beetles (Coleoptera, Carabidae) in forests of the Mordovia State Nature Reserve (Russia). Nat Conserv Res 4 (Suppl.1): 11-20. DOI: $10.24189 /$ ncr.2019.009 
Ruchin AB, Egorov LV, Semishin GB. 2019b. Ladybird beetles fauna (Coleoptera: Coccinellidae) of the Republic of Mordovia, Russia. Biodiversitas 20: 316-327.

Savchenko EN. 1961. Crane-flies (Diptera, Tipulidae), Subfam. Tipulinae, Genus Tipula L., (part 1). Fauna USSR, N.S. 79, Nasekomye Dvukrylye [Diptera], 2 (3) [Russian]

Savchenko EN. 1964. Crane-flies (Diptera, Tipulidae), Subfam. Tipulinae, Genus Tipula L., 2. Fauna USSR, N.S. 89, Nasekomye Dvukrylye [Diptera], 2 (4). [Russian]

Savchenko EN. 1973. Crane-flies (Fam. Tipulidae), Subfam. Tipulinae and Flabelliferinae. Fauna USSR, N.S. 105, Nasekomye Dvukrylye [Diptera], 2 (5). [Russian]

Savchenko EN. 1983. Crane-flies (Fam. Tipulidae), Introduction, Subfam Dolichopezinae, subfam. Tipulinae (start). Fauna USSR, N.S. 127, Nasekomye Dvukrylye [Diptera], 2 (1-2). [Russian]
Savchenko EN. 1986. Komary-limoniidy [limoniid-flies]. (General description, subfamilies Pediciinae and Hexatominae). Fauna Ukrainy 14 (2):-. [Russian]

Savchenko EN. 1989. Komary-limoniidy fauny SSSR [Limoniid-flies fauna of the USSR]. Naukova Dumka, Kiev. [Russian]

Timraleev ZA. 1992. Harmful and useful insects of grain crops of the South of the non-Chernozem zone of Russia. Saransk. [Russian]

Tomaszewska W, Egorov LV, Ruchin AB, Vlasov DV. 2018. First record of Clemmus troglodytes (Coleoptera: Coccinelloidea, Anamorphidae) for the fauna of Russia. Nat Conserv Res 3 (3): 103-105. DOI: $10.24189 /$ ncr.2018.016

Yamashkin AA. 1998. Physical and Geographical Conditions of the Republic Mordovia. Publisher of the Mordovian State University, Saransk. [Russian] 